\title{
Review on Patient-Cooperative Control Strategies for Upper-Limb Rehabilitation Exoskeletons
}

\author{
Stefano Dalla Gasperina ${ }^{1,2 *}$, Loris Roveda ${ }^{3}$, Alessandra Pedrocchi ${ }^{1,2}$, Francesco Braghin $^{2,4}$ \\ and Marta Gandolla ${ }^{2,4}$
}

${ }^{1}$ NearLab, Department of Electronics, Information and Bioengineering, Politecnico di Milano, Milan, Italy, ${ }^{2}$ WE-COBOT Lab, Polo Territoriale di Lecco, Politecnico di Milano, Lecco, Italy, ${ }^{3}$ Istituto Dalle Molle di studi sull'Intelligenza Artificiale (IDSIA), USI-SUPSI, Lugano, Switzerland, ${ }^{4}$ Department of Mechanical Engineering, Politecnico di Milano, Milan, Italy

\section{OPEN ACCESS}

Edited by:

Noman Naseer,

Air University, Pakistan

Reviewed by:

Yang Shen,

UBTECH North America Research and Development Center,

United States

Reza Langari,

Texas A\&M University, United States Usman Ghafoor, Institute of Space Technology,

Pakistan

${ }^{*}$ Correspondence:

Stefano Dalla Gasperina

stefano.dallagasperina@polimi.it

Specialty section: This article was submitted to

Biomedical Robotics,

a section of the journal

Frontiers in Robotics and Al

Received: 21 July 2021 Accepted: 25 October 2021 Published: 07 December 2021

Citation:

Dalla Gasperina S, Roveda L,

Pedrocchi A, Braghin F and Gandolla M (2021) Review on Patient-

Cooperative Control Strategies for

Upper-Limb

Rehabilitation Exoskeletons.

Front. Robot. Al 8:745018.

doi: 10.3389/frobt.2021.745018
Technology-supported rehabilitation therapy for neurological patients has gained increasing interest since the last decades. The literature agrees that the goal of robots should be to induce motor plasticity in subjects undergoing rehabilitation treatment by providing the patients with repetitive, intensive, and task-oriented treatment. As a key element, robot controllers should adapt to patients' status and recovery stage. Thus, the design of effective training modalities and their hardware implementation play a crucial role in robot-assisted rehabilitation and strongly influence the treatment outcome. The objective of this paper is to provide a multi-disciplinary vision of patient-cooperative control strategies for upper-limb rehabilitation exoskeletons to help researchers bridge the gap between human motor control aspects, desired rehabilitation training modalities, and their hardware implementations. To this aim, we propose a three-level classification based on 1) "high-level" training modalities, 2) "low-level" control strategies, and 3) "hardware-level" implementation. Then, we provide examples of literature upper-limb exoskeletons to show how the three levels of implementation have been combined to obtain a given high-level behavior, which is specifically designed to promote motor relearning during the rehabilitation treatment. Finally, we emphasize the need for the development of compliant control strategies, based on the collaboration between the exoskeleton and the wearer, we report the key findings to promote the desired physical human-robot interaction for neurorehabilitation, and we provide insights and suggestions for future works.

Keywords: upper-limb exoskeletons, rehabilitation robotics, neurorehabilitation, robot control, motor recovery, physical human-robot interaction

\section{INTRODUCTION}

When recovering from a traumatic event affecting the ability to perform everyday tasks, the primary goal is to regain functional movements, both at the lower limbs (e.g., walking) and upper limbs (i.e., interacting with daily-life objects). The recovery of motor functionalities is usually possible and relatively straightforward when the traumatic event has an orthopedic source. Still, it becomes trivial when the traumatic event has a neurological basis, for example, after stroke (Cieza et al., 2020). The outcome of the rehabilitation treatment strongly depends on some general neurophysiological aspects of motor relearning. Studies demonstrated that crucial features are high-intensity treatment, 
repetitive training, involvement and engagement of the patient, and personalization of the therapy according to the user's residual capability (Langhorne et al., 2009). Given the increasing burden of neurorehabilitation for therapists and the healthcare system, exoskeletons have been proposed since the $90 \mathrm{~s}$ as a suitable support for post-stroke rehabilitation. Technology-supported therapy aims to provide post-stroke patients with mechatronic devices that help them perform rehabilitation exercises that can potentially foster motor plasticity and improve motor recovery. The efficacy of robot-supported interventions has been widely investigated with randomized clinical trials (RCT) as compared to conventional therapy, and scientific literature reports controversial results (Mehrholz, 2019; Rodgers et al., 2019). Instead, recent systematic reviews and meta-analyses confirmed the suitability of the approach to help patients and therapists during the treatment, showing that the use of robotic devices can positively affect the recovery of arm function in patients with stroke (Bertani et al., 2017; Veerbeek et al., 2017; Wu et al., 2021). A focus on the clinical outcomes of robotassisted rehabilitation is not the aim of this paper. However, looking at the characteristics of a successful rehabilitation program, if well designed, exoskeletons can provide highintensity treatment and repetitive training. When coming to the direct involvement of the patient in the control loop (or human-robot interaction strategy) and the personalization of the therapy according to the user's residual capability, these are important key features, which are still under investigation by the scientific community. Overall, robot-mediated rehabilitation therapy should mimic the quality of conventional therapy performed by physiotherapists and assist patients in regaining lost functions through a wide selection of training modalities. Moreover, it should adapt to patients' status and recovery stage, both throughout the single movement and over the rehabilitation treatment (Marchal-Crespo and Reinkensmeyer, 2009). In addition, there is a great effort in the scientific community to develop frameworks that take advantage of non-invasive and portable brain monitor techniques (e.g., EEG Noda et al. (2012); Nicolas-Alonso and Gomez-Gil (2012), fNIRS Hong et al. (2020); Khan et al. (2021)). Such approaches are employed to detect user intention (i.e., brain-machine interface) and to directly evaluate motor recovery in terms of neural plasticity, making the framework even more complex. In this work, we will concentrate on upper limbs recovery and assistance, focusing on control solutions for upper-limb exoskeletons-based on physical human-robot interaction-and their hardware implementation.

\subsection{Upper-Limb Exoskeletons and Human-Robot Interaction}

Upper-limb exoskeletons for rehabilitation have been developed to guide patients in accomplishing functional tasks as human-like as possible to foster brain plasticity towards recovery. Exoskeleton solutions that actively guide motion usually consist of serialconnected links that are actuated by powered joints. The exoskeleton and the user are interconnected through one or more interaction ports, generally represented by ergonomic cuffs. At the interaction ports level, the exoskeleton and the user exchange forces and torques. The process by which the human and the robot interact and exchange effort is usually referred to as physical human-robot interaction. First-generation devices were characterized by rigid movements of human segments along a prescribed trajectory, thus resulting in the exoskeleton applying forces/torques at the interaction ports to guide the motion, independently from the effort generated by the user. Thus, one of the critical advancements in robot-assisted research is describing and harmonizing the relationship between voluntary human activity and robot assistance. In fact, robotassisted movements involved during rehabilitation are characterized by two interactive processes, for which we propose the outline represented in Figure 1. The first process consists of the patient that is encouraged and tries to perform a functional movement, while the latter regards the robot (or the therapist) applying external forces to the patient's arm to assist and correct the movement (Kahn et al., 2006a).

To complete a functional task, from the human physiological perspective, the intention of the movement is elaborated by the Central Nervous System (CNS), which is in charge of delivering appropriate messages to manage movement execution through its actuating port, and namely the muscles. During movement execution, visual and somatosensory systems provide feedbacks that the CNS analyzes to adjust and correct the strategy according to the comparison between the original intention and the effectively executed movement. The motor control theory is itself an active field of research, and there is discussion whether this comparison is performed accordingly to errors detected at the somatosensory (Gandolla et al., 2014) or kinematic level (Krakauer, 2006), which are two sides of the same coin. Similarly, by mimicking the human motor control scheme, the exoskeleton controller cooperates with the human by superimposing to the muscular effort the (external) robotic contribution, and by shaping the relationship between the human motion and the robot assistance. Regardless of the selected control strategy, the aim is to support the desired motion as revealed by physical human-robot interaction. The control scheme corrects for kinematic or dynamic errors and modulates the set-point signals operated by the mechatronic system's actuators. During the motion, the muscle-generated torque $\left(\tau_{\text {muscle }}\right)$ interacts with the actuator-generated torque $\left(\tau_{\text {exo }}\right)$, leading to an interactive human-robot coupled system.

\subsection{Related Works}

The rehabilitation process can be divided into three main stages according to time past from the traumatic event, namely acute, sub-acute and chronic phases (Proietti et al., 2016). Generally, the acute phase refers to the first week(s) after the injury. The subacute phase includes the range between 15-30 and 180 days after the initial stroke (Péter et al., 2011). The chronic phase is instead defined as the open-ended period starting at about 180 days after initial stroke and characterized by generally slow or no clinical progress (Bernhardt et al., 2017). During these phases, the rehabilitation treatment should make the patient progressively regain the range of motion and muscular strength of the injured limb, and the robot-mediated control strategy should adapt 


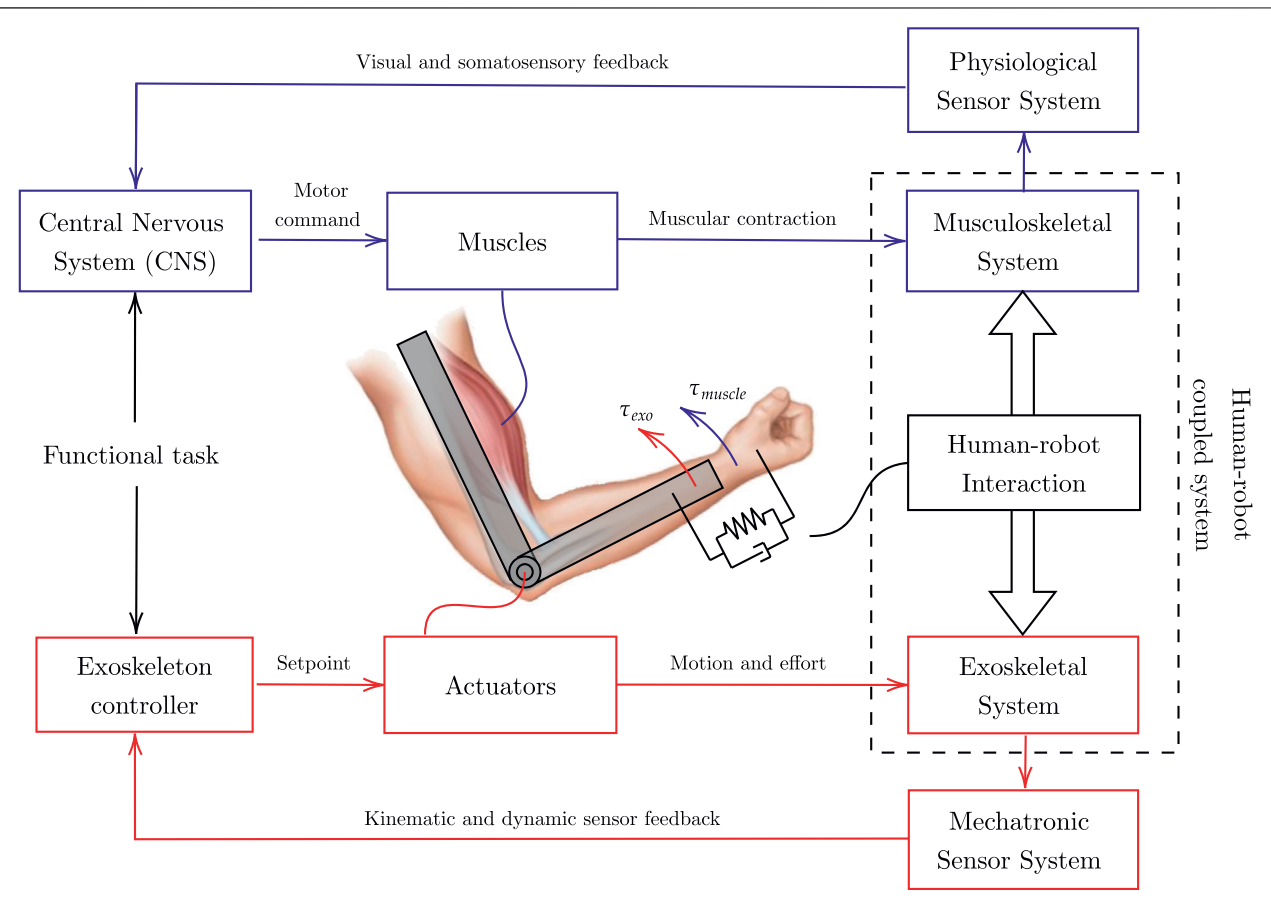

FIGURE 1 | Human-robot interaction representation. The blue scheme represents human motor control, and the red scheme refers to the exoskeleton control. The human-robot coupled system cooperates towards the completion of a shared functional task.

accordingly. In particular, in the earliest stage, since the patient has lost most of the arm functionalities, the robot should help the patient track a predefined trajectory to improve the limb range of motion and reduce muscular atrophy or tendon retractions. Recent studies demonstrated that patients undergoing early robot-mediated therapy within the first weeks after the trauma could gain greater reductions in motor impairment and improvements in functional recovery of the upper-limb (Masiero et al., 2007). As soon as the patient has regained some voluntary muscular contractions, but the generated strength is not adequate to perform precise and complete movements and consequently not sufficient to fulfill functional tasks, the robot should provide the assistance needed to complete the movement, as a physical therapist would do. Moreover, to engage the patient and better induce neural plasticity, the robot should encourage the users to initiate the movements with their active muscular efforts and progressively provide decreasing assistance until the patient has regained the lost functionalities. In fact, it has been demonstrated that the carryover effect is selectively obtained when the patient program the movement and perceives the external assistance as a part of their control loop (Gandolla et al., 2016b). Finally, when stroke survivors have regained most of the range of motion they could recover, the robot should help them recover muscle strength. Recent works demonstrated that improvement in motor function was possible even at late chronic stages, i.e., after the 3-6 months critical window (Ballester et al., 2019; Gandolla et al., 2021). In this situation, the patient actively performs the exercises against resistive forces provided by the robot. Further, challenging strategies can be used to involve and engage the users to continue the rehabilitation treatment. There also exists a branch of robot-assisted rehabilitation that involves other therapeutic approaches combined with upper-limb exoskeletons. For example, Functional Electrical Stimulation (FES) has been used to enhance functional recovery of the paretic arm in stroke survivors (Howlett et al., 2015). The action of FES, combined with the residual voluntary effort of the user, has proven to enhance cortical plasticity (Gandolla et al., 2016b). For example, Ambrosini et al. (2021) demonstrated that EMG-triggered FES combined with anti-gravity robotic assistance could improve the therapeutic effects post-stroke rehabilitation. However, these approaches involve a third interactive process, i.e., the FES-induced muscular contraction, that must be integrated with the robot controller and the user's voluntary actions. For this reason, we will not include in detail FES-based robot-mediated rehabilitation in this work. Overall, it is clear that the design of effective training modalities plays a crucial role in robot-assisted rehabilitation and strongly influences the treatment outcome.

While several reviews on upper-limb exoskeletons are available, most of them deal with the mechanical design of the robotic systems Lo and Xie (2012); Van Delden et al. (2012); Brackenridge et al. (2016); Iandolo et al. (2019); Gull et al. (2020) or with their efficacy in clinical practice Maciejasz et al. (2014); Rehmat et al. (2018). Other reviews investigate robot-mediated rehabilitation control strategies, but they propose taxonomies and classification that are not consistent, and they typically present control methods at high-level of implementation Marchal-Crespo and Reinkensmeyer (2009); Basteris et al. (2014); Proietti et al. (2016); Miao et al. (2018). In particular, with "high-level" 
strategies, the literature usually refers to those control methods that shape the human-robot interaction behavior and focus on specific training modalities.

For instance, Marchal-Crespo and colleagues presented a review on robotic training strategies Marchal-Crespo and Reinkensmeyer (2009). The authors specifically target the review to "high-level" strategies, i.e., such "aspects of the control algorithm that are explicitly designed to provoke motor plasticity". Their work mainly focuses on assistive controllers classified as 1) impedance-based, 2) counterbalance, and 3) EMG-based methods. According to the authors, the impedance-based controllers create restoring forces when the participant deviates from the desired exercise trajectory, but they do not intervene if the subject is moving along the desired path. Counterbalancing controllers, instead, provide weight compensation to the upper-limb through passive elastic elements or active control schemes, but they do not help the participant follow the task trajectory. Finally, EMG-based controllers involve surface electromyography signals (sEMG), and they are aimed at enhancing the residual muscular torques of the participant.

In a different recent systematic review, Basteris et al. (2014) focused on training modalities in robot-mediated upper-limb rehabilitation and they proposed a classification framework based on the expected subject's status during human-robot interaction. In their work, training modalities are divided in four macro categories: 1) active, 2) active-assistive, 3) passive, and 3) resistive. In active mode, the robot does not apply force to the subject's limb and behaves compliantly with the user's movements. In active-assistive mode, it provides assistance towards the completion of the task. In contrast, in passive mode, the robot performs the movement without accounting for the subject's activity, while in resistive mode, it provides forces opposed to the movement. The authors also underline that the literature lacks information regarding the implementation of the different modalities by different research groups.

Another example of review regarding upper-limb exoskeleton control strategies has been proposed by Proietti et al. (2016). The authors presented a taxonomy based on three main global rehabilitation features: 1) assistance, 2) correction, and 3) resistance. While assistance refers to the ability of the robot to support the weight of the limb and provide forces to complete the task, with correction strategies, the robot does not assist the patient, but it corrects the movement to follow a desired path and to provide coordination among joints. Finally, resistance concerns the robot acting against the desired movement. However, the authors state that such features are often combined to properly render the desired human-robot interaction.

\subsection{Aim of the Review}

At this stage, it is clear that different research groups presented different taxonomies and classifications, which are not consistent among works. One of the most challenging aspects of reviewing control strategies for rehabilitation exoskeletons is to provide the understanding of the control method, which most of the time is embodied in nested control algorithms and strongly depends on the available robot hardware. In fact, none of the reviews presented in the literature spans from "high-level" training modalities, to "low-level" control scheme implementation, to "hardware-level" implementation, to the characterization of the needed sensor systems, and they do not provide a match between these aspects. The objective of this review is to provide a multi-disciplinary vision of patient-cooperative control algorithms for upper-limb rehabilitation exoskeletons. The aim is to bridge the gap between human motor control aspects, rehabilitation training modalities, and robot development. To this aim, we propose a three-level classification (Table 1). The first level deals with literature high-level human-robot interaction training modalities, which directly relate to the desired behavior of the rehabilitation exercise and to the capability of the robotic exoskeleton to induce motor recovery according to the patients' status. Such high-level modalities are in turn embodied by lowlevel control strategies, which promote a large variety of physical human-robot interaction according to the residual capabilities of the user. Thus, in the second level, we focus on low-level control schemes that are exploited to promote compliant motion and to display the desired human-robot behavior. Instead, in the third level, namely hardware-level, we draw some insights regarding the state-of-the-art hardware implementation, mainly focusing on actuation, transmission and sensor system technologies. Finally, we outline how different research groups could achieve the desired physical human-robot interaction with their developed hardware. To this aim, we review some upperlimb exoskeleton works as examples of possible different choices made at the three proposed levels. Indeed, to promote the desired human-robot interaction behavior, different approaches can be followed at different levels of implementation.

\section{HIGH-LEVEL REHABILITATION TRAINING MODALITIES}

High-level training modalities have been proposed to promote motor recovery at different stages of the rehabilitation treatment, taking inspiration from neuroplasticity and neurophysiological aspects that are explicitly involved during motor relearning after stroke (Krakauer, 2006; Reinkensmeyer et al., 2016). What researchers want to achieve is to maximize the outcome of the rehabilitation by actively involving the patient in the process and by minimizing the robot effort needed for the completion of the rehabilitation task. To cope with this objective, the robots should cooperate with the subjects during the treatment as a therapist would do. High-level training modalities are usually classified according to the physical interaction between the subject and the robot during the rehabilitation training. Thus, most researchers relate rehabilitation modalities to the subject's status and engagement (Marchal-Crespo and Reinkensmeyer, 2009; Basteris et al., 2014; Trigili et al., 2019), others to the robot's behavior (Pirondini et al., 2016). However, each research group presents a different classification, which leads to non-coherent and misaligned literature taxonomy. In this review, we posit that upper-limb exoskeletons for rehabilitation can mainly operate in four macro-modalities: 1) passive, 2) active-assistive, 3) active, 
TABLE 1 | Presented classification of control methods for patient-cooperative compliant robotics for upper-limb rehabilitation.

Term

"High-level" training modalities

"Low-level" control strategies

\section{Description}

Control strategy that does not necessarily depend on the developed hardware. Directly relates to the desired human-robot interaction behavior during the rehabilitation exercise. Explicitly designed to induce motor plasticity according to the stage of the recover process, and to improve the treatment outcome

Control strategy that depends on the developed hardware. Baseline control law that represents a substrate for implementing a variety of "high-level" modalities. Relates to the capability to promote shared, cooperative, compliant motion between the subject and the robot

Hardware implementation and control approaches used to promote transparency and compliant motion. Relates to actuation, transmission and sensor technologies involved in the development of compliant joints for rehabilitation exoskeletons

TABLE 2 | High-level training modalities for upper-limb robot-mediated rehabilitation. Classification refers to subject's status at interaction. Red arrows represent exoskeleton assistance (solid) or resistance (dashed). Blue arrows indicate user voluntary effort, if present.

\begin{tabular}{|c|c|c|c|c|}
\hline $\begin{array}{l}\text { High-level } \\
\text { modalities }\end{array}$ & Passive & Active-assistive & Active & Resistive \\
\hline Features & $\begin{array}{l}\text { The robot performs the task without } \\
\text { accounting for subject's effort. The } \\
\text { robot corrects trajectory errors }\end{array}$ & $\begin{array}{l}\text { The robot and the subject perform the } \\
\text { task cooperatively. The robot can } \\
\text { provide weight counterbalance or } \\
\text { trajectory-based corrective } \\
\text { assistance }\end{array}$ & $\begin{array}{l}\text { The subject actively performs the } \\
\text { task. The robot does not provide } \\
\text { assistance nor resistance to the } \\
\text { subject. No time-dependent } \\
\text { trajectory is present }\end{array}$ & $\begin{array}{l}\text { The subject actively performs the } \\
\text { task. The robot resists to the } \\
\text { movement by providing opposing } \\
\text { forces }\end{array}$ \\
\hline
\end{tabular}

\begin{abstract}
Human-
robot
\end{abstract}

interaction

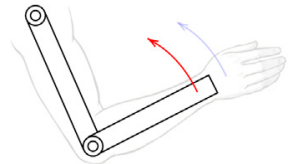

Rationale

Prevents soft tissue stiffening. Passive mobilization generates somatosensory stimulation

Variants
Passive-triggered strategies, Teach-and-replay strategies, Passive-mirrored strategies

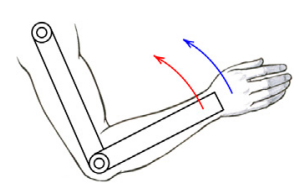

Preserves subject motivation and self-esteem. Subject's involvement promotes motor learning

Purely corrective and weight counterbalance assistance, Inter-joint coordination assistance, Adaptive assistance

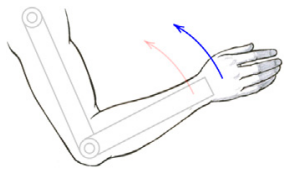

The robot is a measurement device. Permits ROM exploration and do not limit subject's voluntary free movements

Tunneling or trajectory-constrained Viscous-field and errorstrategies

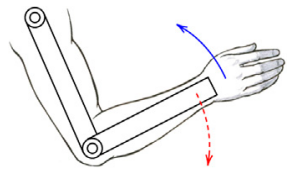

Promotes subject's involvement and potentiate muscular strength augmentation strategies and 4) resistive, according to the human-robot interaction behavior, which are summarized in Table 2.

\subsection{Passive Modalities}

One of the first approaches used in neurorehabilitation regards passive mobilization of the patient's limb along a desired trajectory (Lum et al., 2006). The term "passive" refers to the subject's interaction status, by which the exoskeleton is "active" and performs the movement without accounting for the subject's intention of action. The robot provides stiff behavior and applies high corrective forces to follow the desired trajectory (Marchal-Crespo and Reinkensmeyer, 2009). However, passive mobilization has been proven to limit one of the most important mechanisms of motor relearning: it prevents participants to program in advance the movement, thus it limits the capability to learn from their mistakes, which are driving signals for motor learning (Shadmehr et al., 2010). In fact, the CNS creates an internal model of the environmental dynamics and, during human motor adaptation, it learns to anticipate the movement according to somatosensory and kinematic errors. (Patton et al., 2006; Emken et al., 2007b). In a clinical setting, passive mobilization is usually only operated during the first stages of motor recovery. The rationale of early mobilization is that passive stretching of the limb can prevent stiffening of soft tissue and it helps to reduce spasticity and tendon retractions (Masiero et al., 2007). Moreover, repetitive movements of the limb can generate somatosensory stimulation that can potentially induce brain plasticity and help patients re-learn the desired muscular activation patterns (Bastian, 2008; Crespo and Reinkensmeyer, 2008). Different variants of passive mode are present in literature.

\subsubsection{Passive-Triggered Mode}

The passive-triggered mode consists in the wearer that triggers the exoskeleton assistance as in passive mode (Proietti et al., 2016). This encourages the participant to self-initiate movements, which is an essential feature for motor relearning (MarchalCrespo and Reinkensmeyer, 2009). The trigger can derive from both cognitive or physical human-machine interfaces. On the one hand, participants can initiate the movement by 
means of movement intention detection that can be performed by means of gaze-tracking systems (Frisoli et al., 2012; Novak and Riener, 2013), Motor Imagery based Brain Computer Interface (MI-BCI) (Barsotti et al., 2015; Brauchle et al., 2015), or tonguebased interfaces (Ostadabbas et al., 2016). Alternatively, the passive assistance can be triggered by allowing the participants to attempt a movement with their residual muscular force (i.e., without any robotic support) and initiate the movement after some performance conditions are met. In particular, the movement can be triggered by spatial trajectory tracking errors (Kahn et al., 2006b), movement speed (Krebs et al., 2003), residual forces of the participant (Colombo et al., 2005; Chang et al., 2007) or EMG-based intention detection (Dipietro et al., 2005; Gandolla et al., 2016a). We underline that the triggered assistance is generally applied to passive mobilization of the arm, but it can be also applied to controllers that apply different levels of assistance and resistance to support the arm motion, such as active-assistive controllers.

\subsubsection{Teach-and-Replay Mode}

Different methods exist to define the reference trajectories to be followed by the robot in passive mode. In teach-and-replay mode, joint trajectories are created by recording the robot joint angles during a teaching phase. In this phase, the robot is generally operated in transparent mode (the controller compensates for the robot weight and dynamics) not to resist external forces and to undergo external motion. The therapist guides the affected arm in the workspace, and the desired trajectory is recorded from the exoskeleton joints. In some approaches, relevant way-points are determined, and the trajectory is optimized through a minimumjerk algorithm to avoid undesired oscillations and achieve natural human-like movements (Nef et al., 2007; Lin et al., 2021). Then, the robot actively performs the task taught by the therapist, replays the joint trajectories, and corrects trajectory deviations with corrective gains (Kumar et al., 2019). The therapist can usually tune the execution velocity of the task to match the patient's needs (Nef et al., 2007; Xu et al., 2011). When the desired movement is registered apriori by the contralateral arm (i.e., the healthy one), this modality can also be addressed as record-and-replay mode (Proietti et al., 2016).

\subsubsection{Passive-Mirrored Mode}

A different option is the passive-mirrored mode, which can be implemented only with exoskeletons provided with two arms (Van Delden et al., 2012). The strategy consists of passively mimicking the behavior of the healthy limb by supporting the impaired one passively (Proietti et al., 2016). Usually, this mode can also be referred to as "master-slave" mode since the desired trajectory is continuously computed and commanded by its contralateral side, which is generally operated to behave transparently to the healthy limb (Colizzi et al., 2009; Kumar et al., 2019).

\subsection{Active-Assistive Modalities}

Since passive controllers do not involve active participation from the patient, the literature suggests that more complex control strategies based on subject's involvement could lead to better results, at least after the first stages of the rehabilitation process (Huang and Krakauer, 2009; Reinkensmeyer et al., 2016). This is the case of assistive controllers, by which participants are involved in the completion of the task, while the robot partially assist them in the completion of the task. Due to their nature, assistive strategies guarantee compliant interaction between the human and the robot, and they permit deviation from the desired trajectory (if it exists). As previously mentioned, this feature is a key concept for motor learning as it preserves patients motivation and self-esteem while forcing them to actively adapt their internal models to minimize kinematic tracking errors (Krakauer, 2006; Shadmehr et al., 2010). Similarly to what introduced by Marchal-Crespo and Reinkensmeyer (2009), we further divide active-assistive modalities in two different groups: 1) weight counterbalance assistance, which introduces an offset compensation that counterbalances the weight of the arm; 2) trajectory-based corrective assistance, which generates a force-field environment that helps the user follow the desired trajectory, and 3) inter-joints coordination assistance, which regulates the coordination of the joints and promotes physiological synergies during trajectory-based and free movements. From a more control-based perspective, active-assistive modalities can thus be implemented through a feedback loop combined with a feedforward contribution, as shown in Figure 2. The feedback closed-loop regulates the position or the interaction forces along the reference exercise trajectory (i.e., impedance-based correction), while the feedforward loop compensates for perturbation with a modelbased prediction, such as weight counterbalance assistance and friction compensation.

On top of this general control scheme, several additional features can be added to achieve inter-joint coordination, to implement mirror-based or teach-and-play strategies, or to adapt the assistance according to the treatment outcome. Most exoskeleton prototypes can be operated by a combination of these features.

\subsubsection{Weight Counterbalance Assistance}

In the first case the robot provides the effort only to compensate for weak muscular tone that is unable to support the weight of the arm. In purely counterbalancing strategies, there is no trajectory tracking correction, and the user can actively explore the range of motion. Weight counterbalance is usually implemented through feedforward compensation of the arm weight and dynamics. Several anti-gravity compensation algorithms are available in literature. Most methods are based on dynamic models of the robot-patient system (Just et al., 2017). To compensate for the robot dynamics, its mechanical properties (masses, centers of mass and inertia tensors of each joint-link) are usually extracted from the CAD model of the robotic system (Nef et al., 2007; Just et al., 2016), while weights and lengths of the human arm can be derived from literature anthropometric tables, such as (Winter, 2009). Once the dynamic properties of the two interacting systems are obtained, they are fed in to geometric (Moubarak et al., 2010), Lagrangian (Nef et al., 2007) or recursive (Kim and Deshpande, 2017) inverse-dynamics algorithms to compute the desired joint torques to compensate for the gravity of the 


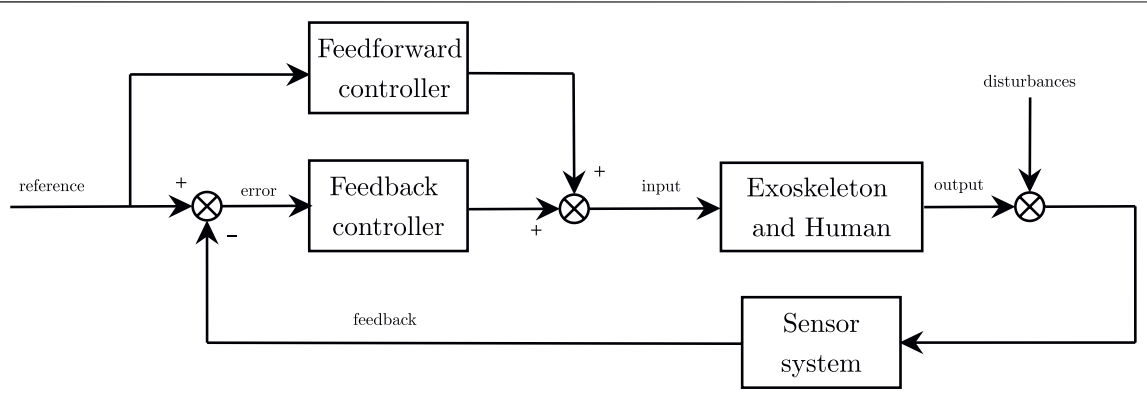

FIGURE 2 | General control scheme. Feedback control (impedance-based corrective assistance) and feedforward control (counterbalance assistance) sum up to compute the desired control input.

human-robot system. However, mathematical models do not always entail a real experience of weight relief for the enduser, and methods to compensate for inertia and load uncertainties have been developed for safe and accurate control of upper-limb exoskeletons. For instance, Wang and Barry (2021) developed a $H_{\infty}$ robust adaptive controller that can adapt to the inertia and load uncertainties and compensate for their effects. In a simulation study, the authors proved that such adaptive controllers could be applied to safe and reliable motion control of rehabilitation exoskeletons. Other approaches are instead based on measurements from force-interfaces (Ragonesi et al., 2013; Just et al., 2020), and combine the experimental data to accurately identify the gravity term without extracting mass and inertia parameters (Moubarak et al., 2010).

Yet another solution to the weight balancing problem is using passive elastic elements to generate additional torques to counterbalance gravity. For instance, the RETRAINER (Ambrosini et al., 2017; Puchinger et al., 2018) employs passive springs to compensate for the wearer weight. Other examples, such as the Pneu-WREX (Sanchez et al., 2005; Wolbrecht et al., 2008) or the BLUE SABINO exoskeletons (Perry et al., 2018; Hill et al., 2019), employ elastic elements in combination with active controllers.

\subsubsection{Trajectory-Based Corrective Assistance}

In trajectory-based corrective strategies, the user has to follow a desired trajectory and the robot corrects undesired behavior, similarly to a position control system, but with a more compliant behavior (Marchal-Crespo and Reinkensmeyer, 2009). The exoskeleton usually does not intervene as long as the patient is following the correct movement. In fact, to accommodate human variability in performing movement, a deadband is usually introduced where the user can move freely. Outside the deadband, if the subject deviates from the target trajectory, the system produces a gradient of restoring forces that usually vary proportional to the trajectory deviation (Nef et al., 2007). Despite the low-level control strategy, the robot is usually commanded to recover from kinematic errors through a virtual zeroth order impedance (i.e., a spring), namely implementing pure stiffness control. The controller implements a corrective action or force-field to guide the user along a desired trajectory or path. By relaxing the corrective gains of the exoskeleton (i.e., by lowering the virtual stiffness), the system displays a more compliant behavior. More recent upperlimb exoskeletons include also corrective controllers provided with viscous force-fields that dampen and stabilize the movements (Proietti et al., 2015; Kim and Deshpande, 2017).

\subsubsection{Inter-joint Coordination Assistance}

Jarrassé et al. (2014) presented a review of studies on upper-limb coordination in stroke patients, intending to illustrate the potential of robotic exoskeletons to rehabilitate inter-joint coordination. Usually, inter-joint coordination can be addressed as a kinematic problem that promotes the activation of physiological muscular synergies compromised by the stroke event. However, most training strategies focus on supporting all the joints of the exoskeletons independently. Very few approaches have attempted to address the spatio-temporal relationship between joints, and the clinical efficacy of this approach is still questionable (Jarrassé et al., 2014). Since most active-assistive controllers follow a reference trajectory, one of the simplest ways to promote inter-joint coordination is to generate a set of joint trajectories that respect specific coordination and timedependency among them. However, computing such joint trajectories is a significant issue. They can be recorded from physiological movements performed by healthy subjects, or the therapist can guide them in a teach-and-replay fashion, or they can be computed through optimal inter-joint coordination inverse-kinematics planners. However, these approaches still require programming specific movements in advance and need to be re-computed for each task or exercise. Consequently, they limit the patients' freedom of movement with the exoskeleton, and they do not investigate the inter-joint coordination problem as a whole.

For instance, Brokaw et al. (2013) developed a Time Independent Functional Training (TIFT) method that provides focused training of inter-joint coordination after stroke and permits movement only if a good level of coordination is achieved. In detail, TIFT provides joint-space walls that resist movement patterns that are inconsistent with the targeted shoulder-elbow inter-joint coordination pattern. Time independence is added to promote voluntary motion from the user without constraining the patient's arm to a fixed, rigid 
trajectory. Similarly, Crocher et al. (2010) proposed a controller which allows to impose velocity-based coordination through viscous force-field without constraining end-point motion. Specifically, the controller does not impose any trajectory, but it reacts user-applied forces by generating joint torques that restrict the motion when a certain velocity-based inter-joint coordination is not obtained. The same approach was used to perturb the human natural inter-joint coordination in healthy subjects Proietti et al. (2017). Results showed that the controller did not directly constrain end-effector movements, but it applied inter-joint velocity-dependent perturbing force fields distributed at the joint-level that disturbed the users' natural upper-limb coordination strategy.

Instead, besides the existence or not of a pre-defined desired trajectory, Kim and Deshpande (2015) presented a control strategy for the shoulder mechanism of an upper-body exoskeleton to assist in achieving coordinated motion at the shoulder complex. The idea is to introduce a coupling torque according to an impedance-based control law that adjusts the shoulder scapulohumeral rhythm configuration. The reference position for the shoulder elevation is computed according to an experimentally obtained quadratic law that correlates the shoulder elevation angle to the humerothoracic arm elevation. Such a relationship can be included and actuated both during free-space motion and along with exercise trajectories. In the first case, the controller implements a reactive action that corrects undesired postures with inter-joint coordination torques (Kim and Deshpande, 2017). The user can explore the range of motion using all the exoskeleton joints, and the corrective torques are applied only at certain joints to maintain the desired coupling. In the latter case, a proper inverse-kinematics algorithm includes inter-joint coordination constraints within the optimization problem. The algorithm exploits the kinematic redundancy of the robot (e.g., through the swivel angle) to reconfigure the exoskeleton according to the scapulohumeral rhythm and computes the desired joint trajectories (Dalla Gasperina et al., 2020).

\subsubsection{Assistance Adaptation}

However, to optimize the outcome of motor learning and to avoid the "slacking" effect, the assistance should be tailored to each stroke patient throughout the movements and over the rehabilitation treatment. Namely, the slacking behavior of the human motor control regards the patient that, trying to optimize the effort to accomplish a task, may learn to provide only the strictly sufficient amount of force needed to complete the task and it takes advantage of the exoskeleton assistance, which performs most of the physical effort (Marchal-Crespo and Reinkensmeyer, 2009). To avoid such a phenomena, the assistance should be supplied only when the subject is not able to actively complete the task and tailored to recovery stage. Different approaches for assistance adaptation have been explored in literature. They mainly involve trial-by-trial adaptation to modulate the robot assistance according to some user-specific performance metrics. For example, adapting control parameters is a key aspect of patient-cooperative strategies, by which the assistance can be automatically tailored to the participant's performances and needs. The goal is to keep the users engaged and actively participating to the treatment, by providing the minimum assistance level to fulfill the task and, at the same time, by promoting the maximum achievable patient muscular effort (Proietti et al., 2016). Adaptive assistance strategies are also referenced as assisted-as-needed strategies and are usually governed trial-by-trial through the following general adaptation control law:

$$
u_{i}=f u_{i-1}+g E_{i}
$$

where $u_{i}$ is the assistance (or control parameter) that is adapted over time, $E_{i}$ is a performance error or metric that can denote the capability of the participant to initiate the movement, to follow a desired path, or to reach a target, and $i$ indicates the $i^{\text {th }}$ trial. $f$ is a forgetting factor $(0<f<1)$, included to avoid slacking and to promote continuous involvement of the participant and $g$ is the gain that determines the reaction timing of the adaptation control law. Including the forgetting term is a key feature to challenge the participant, even if performance errors are low. Indeed, if we consider removing the forgetting term (i.e., $f=1$ ), the control parameters can saturate to the configuration that optimizes the performances, without taking into account the participant effort and engagement. According to the previously described taxonomy, adaptation can occur at both feedback and feedforward assistance loops. In the first case, robot stiffness and corrective force-fields are tuned according to the participants' abilities and effort. For instance, Krebs et al. (2003) first proposed a performance-based control algorithm, which tunes the corrective assistance according to speed, time, or EMG signals. Similarly, the correction can be tuned trial-by-trial according to error-based kinematic performance metrics (Proietti et al., 2015; Pérez-Ibarra et al., 2019). For example, the adaptation control law can rely on terms related to the difference between the measured trajectory and the one desired to fulfill the task, the normalized distance from a specific target, or indexes that indicate the accuracy in drawing a geometric shape (Stroppa et al., 2017b, 2018).

Alternatively, the adaptation can be applied to the feedforward assistance, as presented by Wolbrecht et al. (2008). The authors implemented an assist-as-needed controller that adapts the feedforward assistance, which is computed using radial basis functions and learned on subject's abilities. They added an error-based learning factor, which iteratively adapts the feedforward contribution, and a force decay, which reduces the support when the subject is able to perform the movement correctly.

\subsection{Active Modalities}

Active modalities, also known as "transparent" modalities, are characterized by a "human-active/robot-passive" behavior. The robot does not provide assistance, nor resistance to the movement, and the subject is allowed to perform movements without perceiving the robot effort. Active modalities can be beneficial as they enable the exoskeleton to become a measurement device (Nordin et al., 2014). Recent studies demonstrated that kinematic data can bring meaningful 
information to clinical assessment in post-stroke rehabilitation (Bigoni et al., 2016). De Oliveira et al. (2021) demonstrated that exoskeleton joint angle data are accurate measurements of arm and shoulder kinematics. However, when the robots are operated in active mode for assessment purposes, transparency is a fundamental feature. If the robot provides non-zero torque biases while the wearer is being evaluated, it generates undesired resistances during the upper-limb motion of subjects and it can consequently influence the performance and consequently the assessment (Proietti et al., 2016). When the robot is operated in active mode, the range of motion of each joint can be tuned and limited by control to avoid that the wearer overcomes physiological limits. Usually, range of motion boundaries are implemented through virtual walls, which can be implemented through repulsive virtual spring-damper systems.

\subsubsection{Tunneling Strategies}

As we previously described, corrective strategies usually provide assistance to help the subject follow the desired trajectory both along longitudinal and orthogonal directions. Conversely, the socalled tunneling strategies usually permit free movements, and they provide correction only when boundaries conditions are met. The concept is to create a virtual cylindrical channel at the end-effector that permits free active movements along the longitudinal direction, but restricts movements in radial directions by applying restoring forces to the end-effector position, if the user exits the virtual channel. In order words, tunneling strategies permit active free movement and bound the task-space or joint-space workspace with software virtual walls and boundaries. Since these strategies do not assist the movement along the trajectory main direction, as stated by Proietti et al. (2016), the concept is linked to time-independence of the task references. In particular, in such modalities, there are not trajectory profile references that relate position, velocity, and time. Instead, the controller is fed with a time-independent threedimensional desired path. Guidali et al. (2011) implemented a tunneling strategy by subdividing the task in multiple submovements, and creating force-fields channels to correct the hand position within each sub-movement. Then, after the user had reached a way-point, a trajectory generator algorithm updates the trajectory for the next sub-movement. Similarly, Wu et al. (2018a) implemented a three-dimensional channel based on three concentric channels. The inner channel permits active free movements, the central one assists the user to reach the inner channel, while the outer channel restricts movement directed out of the virtual tunnel. In some works, a timeouttriggered assistance, also known as back-wall, is added to help the users to complete the task when they get stuck and they are not able to actively initiate or finish the movement. The back-wall is usually implemented through a pushing force along the longitudinal direction of the channel (Proietti et al., 2016). If such timeout-triggered assistance is present, tunneling strategies can be become assistive as well. Thus, the taxonomy can be confusing and it can be difficult to distinguish purely tunneling strategies, with or without back-wall, from active-assistive modalities.

\subsection{Resistive and Challenging Modalities}

Historically, rehabilitation robots were designed to assist the patient during the initial phases after stroke, i.e., when the patient is severely impaired and needs substantial assistance to complete functional tasks. Then, when the patient has (hopefully) relearned most of the lost motor functionalities but still has to gain some muscular tone, conventional therapy proposes gymlike body-weight exercises. Resistive modalities have been recently introduced as a rehabilitation solution for the latest stages of the motor recovery process to engage the patients during their progression through robot-mediated exercises. In fact, robots can provide an aquatic therapy-like environment that allows user-driven free movements with or without viscous resistance (Kyoungchul et al., 2010). Usually, resistive modalities do not follow trajectory references. Still, they permit the user to actively explore the workspace, and the exoskeleton resists user's movement through virtual viscous force-fields, which are usually inversely proportional to the movement velocity (Song et al., 2014). Finally, we could include in this category also other challenging strategies based on error-augmentation methods since they indirectly resist the motion by repressing the voluntary movement or by emphasizing kinematics errors. Error-augmentation consists of algorithms that, through repulsive forces, amplify movement errors rather than decrease them (Patton et al., 2006). Indeed, as previously mentioned, motor learning has underlined that kinematic errors are fundamental neural signals to improve motor adaptation (Emken et al., 2007a). A similar approach involves instead the implementation of task-space force fields that push the user's arm away from equilibrium points or comfortable positions to enhance workspace exploration (Wright et al., 2015, 2018). Resistive and challenging modalities have been broadly investigated in gait and locomotion analysis. However, few studies have been performed in upper-limb functional rehabilitation (Abdollahi et al., 2014; Israely and Carmeli, 2016).

\section{LOW-LEVEL ROBOT-ASSISTED CONTROL STRATEGIES}

To guarantee a good collaboration of subject and exoskeleton during physical human-robot interaction, the robot should display a wide range of haptic mechanical impedance, which should span from high-compliance (low-resistance) to highstiffness behaviors. While achieving rigid control can be considered trivial, promoting the so-called compliant motion, i.e., the robotic device should behave transparently to voluntary human activity, can be challenging. Furthermore, its performances are strongly related to the mechanical design of the actuation unit and thus they depend on the specific hardware implementation. Namely, compliant control refers to the capability of the robotic system to generate movement and, simultaneously, to undergo movement if external forces are applied. On the one side, the robot drives the motion of the limb and corrects for trajectory errors. On the other side, the user applies forces/torques to the robot, which should permit deviations from a defined equilibrium point without 
suppressing the voluntary activity. Since compliant motion doesn't limit in any way any intention of movement of the interacting user, it guarantees one of the most fundamental features for efficient motor recovery and demonstrated to be a fundamental, yet challenging, feature in rehabilitation robotics. To make the processes mentioned above interact smoothly, each of them should be aware of the other's behavior. While the human, thanks to its somatosensory and visual systems, can directly feel and monitor the behavior of the robot, both in terms of interaction forces and perceived movements, the robotic device needs an adequate sensors network to detect the involvement and the intention of movement coming from the user. Indeed, exoskeleton developers can follow different approaches to detect the user's intention of movement, which deeply depend on the implemented hardware.

Recently, Calanca et al. (2016) published a survey that presented the state of the art of compliant control algorithms according to the available sensor networks and control schemes. The authors analyzed solutions from traditional robotics, usually involving stiff joints, to more recent approaches that combine soft joints with advanced control schemes. Indeed, the concept of compliant motion refers to the capability of a system to shape the dynamical relation between motion and torque/forces, instead of independently controlling the joint motion or the joint torques of the robot. Thus, to promote compliant interaction between the human and the robot, along trajectories or in free motion, several low-level control strategies have been proposed (Miao et al., 2018). Most of the compliant controllers, instead of relying on high-gains corrective position control, implement nested control loops that are usually characterized by an inner high-accuracy loop, which guarantees fast response of the robotic system, and an outer "flexible" loop, which includes the human contribution and implements the interaction control. Such approaches mainly rely on two control schemes: Impedance control (force/torque based control) and its dual admittance control (position based control) Ott et al. (2010); Schumacher et al. (2019). However, as we previously introduced, the perceived compliance can be implemented either through compliant controllers, or through mechanical compliance, for example by using soft joints instead of stiff joints (Calanca et al., 2016, 2017; Schumacher et al., 2019). Thus, in this review, we include and discuss position control of soft joints as it can itself promote compliant interaction control.

\subsection{Impedance Control}

Among all, impedance control is one of the most common approaches, and it has been demonstrated to be a very efficient solution for neurorehabilitation (Marchal-Crespo and Reinkensmeyer, 2009; Mehdi and Boubaker, 2012). It implements dynamic control that shapes the desired mechanical impedance through human-robot interaction: a torque/force output is generated from a position input. In this section, we will firstly describe the main features of impedance control applied to a joint of the robot (i.e., in the joint-space), then we will explain its applicability in the Cartesian-space.

Impedance control was first introduced by Hogan (1985), and it is also referred to as force-based position control or equilibrium point control. Indeed, differently from traditional position control, this approach does not aim at precisely tracking trajectories, but it proposes a trade-off between interaction forces and deviation from the reference motion. To promote this behavior, impedance control is characterized by a nested loop architecture. An inner torque-feedback loop implements the transparent behavior and promotes the mechanical compliance (i.e., it "softens" the control). An outer position-feedback loop corrects for trajectory tracking errors by applying forces or torques aimed at the completion of the task (i.e., it "stiffens" the control). Two different variants of the impedance control can be identified. When the actuation unit is inherently back-drivable, the torque control can be implemented through an open-loop control loop (i.e., implicit impedance). In the other cases, a loadcell or an elastic element is exploited in series as a feedback signal for the closed-loop torque control loop (i.e., explicit impedance) (Khalil and Dombre, 2002). Explicit impedance control improves force sensitivity, but can jeopardize the coupled stability of the human-robot system. In fact, high torque-loop control gains can cause stability issues when in contact with hard surfaces (Calanca et al., 2016; Focchi et al., 2016), thus there exists a trade-off between torque fidelity tracking and stability of the impedance controller. The impedance control schemes (implicit and explicit) can be implemented in the joint-space as shown in Figure 3.

The reference or equilibrium joint position is $\theta_{\text {ref, }}$, while the actual position $\theta_{m}$ is usually measured by motor encoders. The torque control signal $\tau$ is usually computed as:

$$
\tau=I(s)\left(\theta_{\text {ref }}-\theta_{m}\right)+\tau_{\text {ref }}
$$

where $I(s)$ is the mechanical impedance model, usually multiplied by the trajectory tracking error, and $\tau_{\text {ref }}$ represents the torque reference, often used to compensate for gravity and friction torques. The actuator block represents the actuator dynamics and converts the control signal $u$ to the desired output. If the explicit impedance control scheme is exploited, $F(s)$ represents the inner torque control loop, which is in charge of making sure that the measured torque output $\left(\tau_{e}\right)$ tracks its reference $\left(\tau+\tau_{\text {ref }}\right)$. The $F(s)$ estimates the target torque of the actuator $(u)$, usually through a Proportional-Integrative-Derivative (PID) controller. The impedance filter $I(s)$ is generally described by a $n^{\text {th }}$ polynomial system that varies according to the order of the virtualized mechanical impedance system. Impedance control of $0^{\text {th }}$ order, also referenced as pure stiffness control (Trigili et al., 2019), is formally equivalent to a proportional (P) position controller, where the gain represents the desired mechanical stiffness $K_{s}$ (Eq. 3).

$$
I(s)=K_{s}
$$

If a $1^{\text {st }}$ order impedance is implemented, the velocity error, namely $\dot{\theta}_{r e f}-\dot{\theta}_{m}$, is multiplied by a target damping coefficient $K_{d}$, which is usually aimed at reducing jerky oscillations and dissipating spring energy. The $1^{\text {st }}$ order impedance control formally corresponds to a proportional-derivative position controller (Eq. 4).

$$
I(s)=K_{s}+s K_{d}
$$




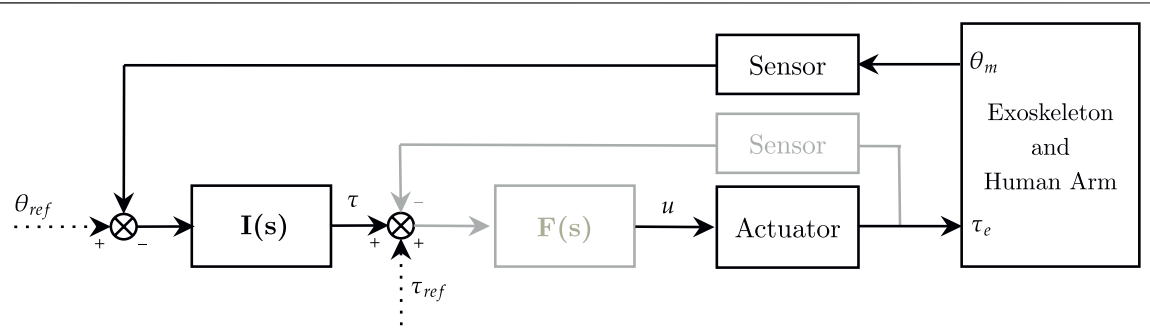

FIGURE 3 | Impedance control scheme in the joint-space. Implicit (black) and explicit (black and gray). I(s) is the impedance controller, $\mathbf{F}(s)$ is the force controller (only explicit). $\theta_{\text {ref }}$ and $\tau_{\text {ref }}$ represent respectively reference angular position and torque, while $u$ refers to the motor corrent control signal.
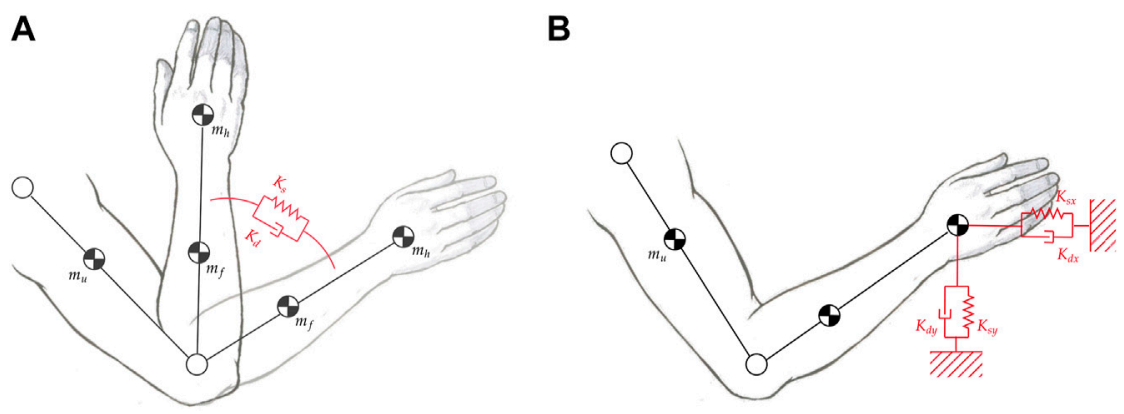

FIGURE 4 | (A) First order impedance model applied at the elbow joint in the joint-space. (B) First order impedance model applied at the elbow joint in the Cartesian-space.

This is one of the most common implementations in rehabilitation robotics as the virtual stiffness, by means of the virtual spring constant $K_{s}$, pulls the joint link towards its reference (i.e., the spring corrects for deviations from the joint trajectory), while the virtual damper $K-d$ dampens oscillations and stabilizes the movement. However, in most cases, since the desired velocity $\dot{\theta}_{\text {ref }}$ is not accessible, the desired velocity can be neglected and set to zero, and the damping coefficient only multiplies the measured velocity. In this way, the damping term is related to the absolute velocity instead of the error velocity, and the controller always provides resistance to the motion, regardless if the user is correctly following or not the desired trajectory (Kim and Deshpande, 2017). In time domain, the control law becomes:

$$
\tau=K_{s}\left(\theta_{r e f}-\theta_{m}\right)+K_{d}\left(\dot{\theta}_{m}\right)+\tau_{r e f}
$$

Finally, impedance control of $2^{\text {nd }}$ order allows to shape also the desired mass/inertia $K_{i}$ of the system. When dealing with rehabilitation robots, usually the desired mechanical inertia is the one of the human arm, thus the second order term can be neglected. The control law becomes as in Eq. 6, which corresponds to a proportional-integral-derivative (PID) velocity controller.

$$
I(s)=K_{s}+s K_{d}+s^{2} K_{i}
$$

Higher order implementations are possible, and the desired impedance can be set to be of arbitrary order. However, if higher orders are concerned, the impedance control parameters would become physically meaningless, and the computation of high order derivatives can introduce noise to the acceleration signals. In this view, first order impedance control is usually preferred.

In rehabilitation robotics, many exoskeletons are controlled in the task-space through Cartesian-space impedance controllers (Frisoli et al., 2009; Nef et al., 2009). This approach is preferred over joint-space impedance control since it favors functional tasks, and it does not require inverse-kinematics algorithms during trajectory generation. The Cartesian-space impedance control scheme is implemented by virtualizing a mechanical impedance in the task-space instead of at the joint level, as shown in Figure 4. While in joint-space the spring-damper system is a rotational system $n$-dimensional ( $n$ represents the number of active degrees-of-freedom of the robot), in Cartesianspace, the mechanical impedance is linear and three-dimensional. In fact, the corrective action is provided by three-dimensional forces, usually referred to as corrective force-fields. Consequently, in order to permit the robot to generate such assistance, there is the need to convert 3-dimensional task-space forces to $n$ dimensional joint-space torques. Generally, the transposed Jacobian matrix is exploited to compute such conversion. The Cartesian-space impedance control scheme is considered a centralized control approach, since it exploits the robot configuration (usually through forward kinematics) to compute the desired torques at each joint, as shown in Figure 5.

In detail, considering a $1^{\text {st }}$ order mechanical impedance on the $x$ Cartesian-direction, the force-fields are computed as: 


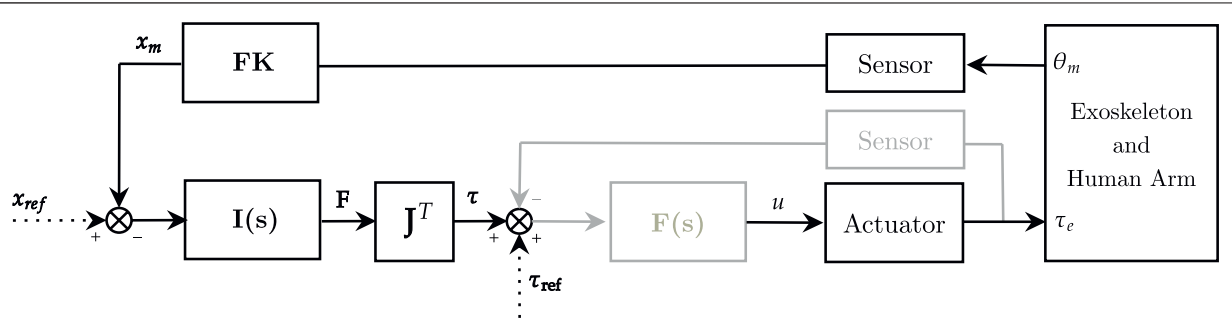

FIGURE 5 | Impedance control scheme in the Cartesian-space. Implicit (black) and explicit (black and gray). I(s) is the impedance controller, $\mathbf{F}(s)$ is the force controller (only explicit). FK represents the forward kinematics model of the exoskeleton, and $\mathbf{J}^{T}$ corresponds to the transposed Jacobian matrix. $\theta_{\text {ref }}$ and $\tau_{\text {ref }}$ represent respectively reference angular position and torque, while $u$ refers to the motor current control signal.

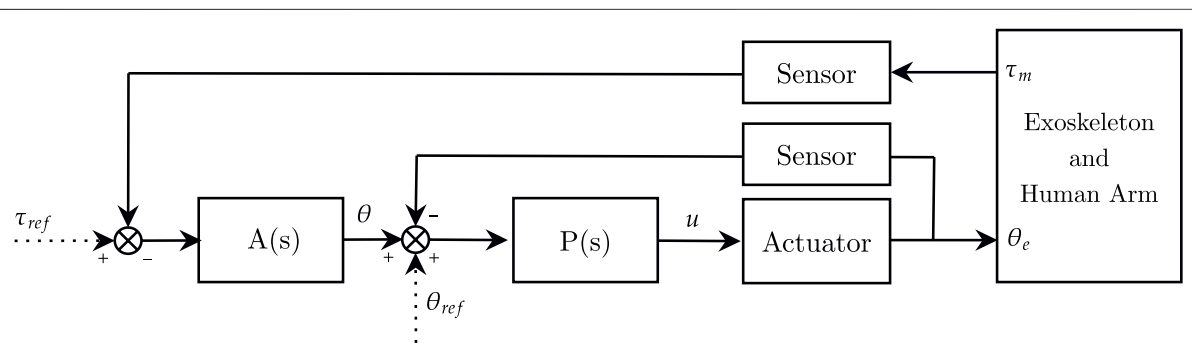

FIGURE 6 | Admittance control scheme in the joint-space. $\mathbf{A}(s)$ is the admittance controller, $\mathbf{P}(s)$ is the position controller. $\theta_{\text {ref }}$ and $\tau_{\text {ref }}$ represent respectively reference angular position and torque, while $u$ refers to the motor current control signal.

$$
F_{x}=K_{s}\left(x_{d e s}-x_{m}\right)-K_{d}\left(\dot{x}_{d e s}-\dot{x}_{m}\right)
$$

where $K_{s}$ and $K_{d}$ are the desired linear spring and damper, respectively, and $x_{m}$ is the measured position of the endeffector, computed through the forward kinematics model of the exoskeleton.

In neurorehabilitation, Cartesian-space impedance controllers can be used to implement tunneling strategies. They permit to discriminate robot assistance along the tangential and orthogonal directions of the end-effector reference motion. Thus, the robot can assist along the axial tunnel direction and correct along the radial direction. Cartesian impedance strategies also intrinsically allow timeindependent relationships among the exoskeleton joints, which is crucial to enable the user to exhibit voluntary movements. In fact, besides the robot configuration, the ultimate goal is to control the pose of the user's hand through spring-damper behavior to follow the desired path. However, this strategy (i.e., Cartesian impedance control) does not control or correct compensatory movements or noncoordination among joints. Therefore, it is more prone to maladaptive plasticity mechanisms. For example, Zhang et al. (2020) presented a novel assisted-as-needed controller that, through a task-space impedance controller, assists the position of the hand of the user to follow a virtual tunnel. Stiffness fields are created to push the end-effector to the center of the tunnel and guide it along the tunnel if the user is not fast enough. Furthermore, the proposed controller can be adjusted through five adjustable parameters to implement different robot-aided rehabilitation training such as passive, active-assistive, active, and resistive training.

\subsection{Admittance Control}

Admittance control is the dual approach to impedance control, and it is generally used as a method to promote physical humanrobot interaction with stiff, non-backdrivable actuators (Keemink et al., 2018). By definition, admittance control actuates motion (usually position or velocity) through a force/torque feedback, and it is also generally known as position-based impedance control or impedance control with force feedback (Ott et al., 2010). The control scheme involves a nested loop architecture, where the inner loop controls the position (or velocity) of the joint, and the outer torque loop computes the motion setpoint according to the desired human-robot interaction, as shown in Figure 6.

In admittance control, the inner loop "stiffens" the joint, and the outer loop "softens" the human-robot interaction behavior (Calanca et al., 2016). Generally, robot weight compensation is not needed since the robot is position controlled. However, in some works, weight compensation is provided in feedforward at the inner position control loop $(\mathbf{P}(s))$ to improve trajectory tracking Bai et al. (2017). The main advantage of using admittance control in rehabilitation robotics is that it does not require intrinsic back-drivability of the actuation unit: the inner motion control loop intrinsically compensates and rejects stiction and dynamic friction. In other words, the outer force loop computes the reference motion that produces a virtual backdrivability of the joint (Calanca et al., 2016). When 
dealing with impedance control, achieving high-fidelity torque control is critical to render a wide variety of mechanical impedance (Z-width), i.e., impedance control requires both high-stiffness gains for good trajectory tracking and lowstiffness gains to promote compliant behavior and its accuracy depends on the capability of the system to deliver high-quality torques. Contrarily, admittance control can exploit the standard features of industrial robots for the implementation of the inner motion loop that can suppress undesired disturbances such as system dynamics and friction, without the need for model-based compensation (Schumacher et al., 2019). The main limitation arises when low-impedance behavior is desired, and highadmittance gains could lead to instability issues. Similar to impedance control, different orders of the admittance filter can be selected. Still, the computation of the reference motion profile in the time-domain may require numerical integration to solve the motion differential equations. In this review, we will consider the admittance model as the relationship between force and position (Ott et al., 2010; Schumacher et al., 2019). In other studies, such as in Calanca et al. (2016), authors described the admittance model as a force-velocity relationship. The admittance model of zero-order is usually referred to as compliance control, and it is formally complementary to stiffness control, by means of the inverse of the desired stiffness $K_{d}$. The desired zero-order admittance is computed as:

$$
\mathbf{A}(s)=1 / K_{d}=K_{d}^{-1}
$$

The $1^{\text {st }}$ order admittance or accommodation control is one of the most common implementations in rehabilitation since it is suitable for slow motion (Keemink et al., 2018). The motion is derived from the force/torque feedback as follows:

$$
\mathbf{A}(s)=1 /\left(K_{d}+s D_{d}\right)=\left(K_{d}+s D_{d}\right)^{-1}
$$

where $D_{d}$ represents the desired impedance damping (or viscous friction).

For example, Zhuang et al. (2019) proposed a first-order admittance model, characterized by a virtual spring-damper interaction system, to control an ankle rehabilitation exoskeleton promoting compliant behavior. By neglecting the zero-order desired stiffness $K_{d}$, the impedance model becomes a pure anti-damping velocity-driven admittance controller, which is generally the simplest way to promote transparent behavior at the joint level.

Finally, the second-order admittance model also permits to shape the desired mass/inertia $M_{d}$ of the virtualized dynamic system. The admittance equation is shown in (Eq. 10).

$$
\mathbf{A}(s)=\frac{1}{\left(K_{d}+s D_{d}+s^{2} M_{d}\right)}=\left(K_{d}+s D_{d}+s^{2} M_{d}\right)^{-1}
$$

The desired stiffness can be removed, and the controller becomes a mass-damper virtualized system, as in Chia et al. (2020).

When coming to rehabilitation exercises, the positioncontrolled trajectories are generally computed as the sum of the desired joint profiles, namely $\theta_{r e f}$, and the angle $\theta$ that is in turn obtained from the admittance model and the interaction forces. In this way, the robot follows the desired movement but permits deviation according to the user's voluntary activity. The reference torque $\tau_{\text {ref }}$ is normally used to filter out gravity effects and static disturbances from the torque/force measurements $\tau_{m}$, but it can also be tuned to include additional external force-fields to the desired physical human-robot interaction. For upper-limb robots, admittance control in the joint-space (i.e., with torque feedback at the joints level) has not been explored yet, since it requires precise mathematical models for gravity compensation. Instead, 6-DOFs force/torque sensors at the interaction ports of the robot are more often exploited to detect the interaction effort with the user. With this approach, there is no need for gravity compensation of the robot (which is position-controlled), and the force feedback does not need additional filtering for gravity effects. Of course, since the force/torque sensors usually detect interaction in the three-dimensional space, a conversion to jointspace motion is needed to feed the inner control loops (that operate at each joint of the robot). If task-space sensors are used, the conversion can be implemented both for the desired position or for the measured feedback. The admittance control scheme for task-space sensors is shown in Figure 7.

With respect to the task/joint space conversion, Wu et al. (2018b) developed a patient-active admittance controlled exoskeleton for upper-limb neurorehabilitation. Interaction forces with the user are acquired with force/torque sensors at the end-effector. The differential desired forces are then fed to a second-order admittance filter that computes the desired differential trajectory in task space. Finally, an inverse kinematics algorithm computes the desired trajectories in the joint-space. Alternatively, Bai et al. (2017) implemented a second-order admittance control scheme with an upper-limb exoskeleton. The forces are measured at the arm cuffs through force-sensing contact sensors. The sensors measure the human-robot interaction forces, which are analyzed by a "force model controller" that computes the desired interaction torque for each joint. Finally, the admittance filter is applied to compute the motion profiles in the joint-space.

As previously mentioned, in admittance control, the intrinsic back-drivability of the exoskeleton joint is not needed. In fact, the robot is position-controlled by the inner loop, which does not guarantee to be intrinsically transparent to user effort, and external signals are used to detect the intention of movement towards a certain direction. Many different approaches are available to detect user-driven movements. The most common approach relies on direct measurement of interaction effort through force/torque sensors at the interface ports of the robot that are usually at the upper arm and forearm cuffs (Kim and Deshpande, 2017; Wu et al., 2018a; Wu et al., 2018b) (Section 4.2). Alternatively, human voluntary effort can be estimated by means of EMG-based sensing (Zhuang et al., 2019), or with forcesensing resistors (FSR) (Bai et al., 2017).

\section{HARDWARE-LEVEL IMPLEMENTATION OF COMPLIANT CONTROL}

When dealing with rehabilitation exoskeletons, most platforms rely on electric motors provided with high-ratio gearboxes to 


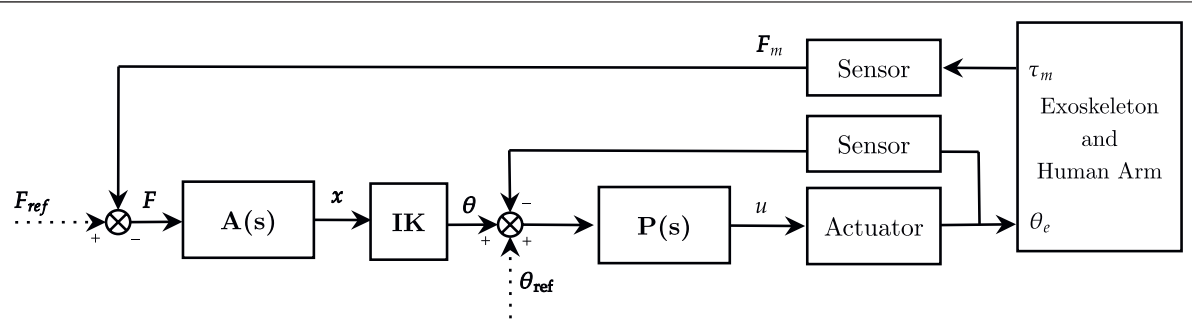

FIGURE 7|Admittance control scheme in the task-space. A(s) is the task-space admittance controller, $\mathbf{P}(s)$ is the joint-space position controller. IK corresponds to the inverse-kinematics algorithm. $\theta_{\text {ref }}$ and $\tau_{\text {ref }}$ represent respectively reference angular position and torque, while $u$ refers to the motor current control signal.

increase the ability to deliver motor torque. However, they are inherently inefficient and they introduce non-linear stiction, static and viscous friction, and reflected inertia, which can compromise back-drivability (Schumacher et al., 2019). Consequently, in most cases the perceived compliance cannot be guaranteed by the mechanical back-drivability of the geared drive itself, and users would need to overcome large torques to initiate voluntary movements (Nef and Lum, 2009). In this review, we focus on electrically powered exoskeletons and we describe three global approaches to promote compliant behavior with geared drives, i.e., to permit the user exert torque onto the robot joint, according to the desired low-level control strategies.

\subsection{Model-Based Compensation}

When dealing with implicit impedance controlled exoskeletons, by which the robot is not provided with additional torque sensors, residual frictional torques need to be compensated by software. A common practice to improve back-drivability of high-ratio gearboxes is to implement friction compensation models (Nef and Lum, 2009; Weiss et al., 2012). In this way, a zero-torque controller could achieve good transparency with low residual resistive torques. Usually, friction is modeled with a kinetic friction term (Coulomb and viscous velocity-dependent) and a breakway friction term, which relates to the stiction phenomena (Armstrong, 1988). The friction compensation is mainly regarded as positive velocity-based feedforward control (Just et al., 2016). Still, the breakaway friction usually cannot be compensated for since the sign of the compensation term depends on the direction of the desired movement, which is not always defined a priori (Nef and Lum, 2009).

\subsection{Interaction Force/Torque Sensing}

Researchers are recently providing their rehabilitation robots and exoskeletons with torque/force sensors, which directly measure the interaction force between the human and the robot (Villani and De Schutter, 2008), namely achieving active compliant control. For impedance control approaches, direct torque sensing permits to implement torque control loops with explicit feedback to reject friction disturbances and to reduce residual resistive torques (Boaventura et al., 2012, 2013). Overall, this approach leads to better torque-tracking performances and improves back-drivability. However, Focchi et al. (2016) demonstrated that high-gain tuning of the torque closed-loop can jeopardize the stability of the robot when touching hard surfaces. Thus, there exists a compromise between low undesired interaction forces and control robustness (Vallery et al., 2008). Torque sensing can also be fed in at the outer loop, such as in admittance control. In this case, the robot is not compliant because of the inner loop, but the effort sensing is used to update the desired trajectory of the inner loop. While torque sensing in impedance control is usually performed in the jointspace, with admittance control, the loadcell is usually installed at the end-effector, through a handle (Ghonasgi et al., 2021), or at the interaction ports, through the arm cuffs that are usually at the upper arm and at the forearm levels (Kim and Deshpande, 2017; Wu et al., 2018a; Kumar et al., 2019). While in most cases the interaction forces are measured with loadcell-based sensors, sometimes force sensing resistors (FSR) are installed inside arm cuffs (Bai et al., 2017).

\subsection{Mechanical Compliance (Series Elastic Actuators)}

While the first two approaches are usually implemented with rigid joints, the perceived compliance can also be implemented by adding mechanical compliance, for example by using soft joints. In fact, compliant control can be also achieved by voluntarily introducing elastic elements (i.e., springs) in series to general purpose electric actuators, namely series elastic actuators (SEA) (Vallery et al., 2008). Several research groups that develop upperlimb exoskeletons are now relying on SEAs (Crea et al., 2016; Chen et al., 2019; Wu et al., 2019) since they are inherently safe, they permit robust force control and they are efficient in periodic tasks (Calanca et al., 2016). Also, if additional mechanical compliance is added to the actuation chain, there is no need for a intrinsic backdrivable geared actuator. However, SEAs, due to the mechanical compliance, result in limited force and position control bandwidth, and can lead to instability issues when trying to achieve high-impedance behavior. Namely, the achievable displayed stiffness of the joint cannot be higher than the physical spring stiffness of the SEA, if passivity is desired (Vallery et al., 2008; Calanca et al., 2017).

The mechanical compliance can potentially simplify the control strategy of the exoskeleton. For example, in Wu et al. (2019); Trigili et al. (2019), compliant control was achieved by position-controlling a SEAs unit. Indeed, there is no need to 
strictly apply compliant control strategies, since the compliance is intrinsically provided by the physical stiffness. Nevertheless, in most cases, a combination of impedance/admittance control of SEA is used to promote variable impedance behavior (Calanca et al., 2016). In some other cases, if the spring stiffness is high enough, the SEA does not provide a perceivable mechanical compliance, but the spring is used only to indirectly compute the joint torque output by measuring its displacement and to dampen high frequency oscillations (Kim and Deshpande, 2017).

Overall, if impedance control strategies are desired, there is the need for good back-drivability of the joint to promote compliant interaction control. Instead, admittance control typically does not require back-drivable joints, and high transmission ratios are preferred to achieve precise position control, but force/torque sensing is mandatory to detect the intention of movement of the user. Finally, if SEA-based joint are designed, compliance is intrinsically promoted, and torque sensing can be achieved with indirect measurements based on the elastic element deformation.

\section{AVAILABLE EXOSKELETON PROTOTYPES}

This section presents and compares some control solutions for arm rehabilitation exoskeleton prototypes available in the literature. The presented list is non-comprehensive, but works were selected to describe and demonstrate how different approaches could promote similar high-level rehabilitation modalities. Indeed, we focused on works that describe how the three levels of implementation have been combined to obtain a given high-level training behavior. For each solution, we explain its functioning at the three levels of the proposed classification, and we report the key findings of each approach.

\subsection{The Aalborg University Exoskeleton}

The exoskeleton described in Bai et al. (2017); Christensen and Bai (2018) is an upper-limb device provided with three actuated DOFs and one passive DoF, developed at the Aalborg University (AAU). The proposed solution addresses the problem related to the design of the shoulder mechanism, allowing the exoskeleton to match the complex motion of the human shoulder joint. The proposed kinematics relies on a spherical mechanism consisting of two revolute joints connected through a double parallelogram linkage. The exoskeleton is equipped with force sensing resistors (FSR) sensors (capable of detecting physical pressure) to measure the interaction between the user and the exoskeleton. Such measurements are elaborated by a force model controller module capable of detecting the direction of the applied interaction forces. Based on the magnitude and direction of the applied forces, the admittance controller has been implemented to provide the subject with the capabilities to operate the exoskeleton based on the applied interaction. Such admittance controller defines the reference velocity for the inner PI trajectory tracking controller with gravity compensation capabilities, allowing to operate the exoskeleton. Preliminary experimental results have shown the performance of the proposed design.

\subsection{The ALEx Exoskeleton}

The Arm Light Exoskeleton (ALEx) is a bimanual robotic device specifically designed for robot-supported rehabilitation of stroke patients. ALEx is a cable-driven, mechanically compliant exoskeleton operated by four actuated and sensorized DOFs (shoulder and elbow) and two passive DOFs (wrist). The robot is equipped with brushless motors located remotely to the exoskeleton joints. The use of compliant cable-based transmission reduces weight and inertia of the system, and the mechanical compliance, introduced by cables, permits to achieve robust force control (Stroppa et al., 2017a). In its first version, ALEx allows to perform movements in three different modalities: 1) robot-passive, namely human-active modality according to our classification (i.e., the participant moves the arm in the workspace and the robot follows the motion), 2) robot-active, namely human-passive modality according to our classification (i.e., the robot guides the participant's arm during the movement), and 3) assisted-as-needed, or passive-triggered modality, by which the robot guides the motion only if a timeout-based trigger is reached (Pirondini et al., 2016). At the low-level, the exoskeleton can be operated in force mode, which provides desired forces at the end-point, or in compliant position mode, which relies on independent position control of the robot compliant joints. For all modalities, the low-level force controller is computed as the sum of several feedforward contributions, including gravity compensation of the moving robotics links, friction compensation of transmission mechanisms, and inertia compensation of moving parts. In a recent study, Stroppa et al. (2017b) presented an adaptive assistance controller based on a Cartesian-space impedance control scheme. The impedance law is based on a massspring-damper dynamic system that corrects joint trajectories in the task-space. Finally, the assist-as-needed paradigm is implemented according to an online performance evaluation of the subject's motor skills.

\subsection{The ARAMIS Exoskeleton}

The ARAMIS exoskeleton is a bi-manual exoskeleton for upperlimb neurorehabilitation after stroke. The robotic platform includes two fully motorized 6-DOFs symmetric exoskeletons (Pignolo et al., 2012). The robot can operate in different modalities that involve the use of both the unaffected and the paretic arm (Pignolo et al., 2016). In synchronous mode, which can be addressed as master-replica mode, the robot supports the impaired limb of the subject and replicates the sample movements performed by the other arm in real-time. In asynchronous mode, instead, the contralateral exoskeleton arm first records a sample movement, then the robot actively supports the paretic arm along the mirrored task. Such trajectories can be recorded either by the patient's unaffected limb or by the therapist's guidance (Dolce et al., 2009). The ARAMIS exoskeleton can also operate in weight counterbalance mode: the robot compensates for the arm weight during movements replicating those executed by the contralateral side (Pignolo et al., 2016). Each joint of the robot is actuated by DC brushed motors coupled with high transmission ratio gearboxes. To enhance the backdrivability of the system, the authors developed an integrated 
joint that relies on a SEA-based design (Colizzi et al., 2009). Series springs are connected at the output shaft of the actuator, and a secondary encoder measures the spring displacement. In this way, the controller can detect whether the patient initiates the movement and the exoskeleton follows the user-driven action.

\subsection{The ARMin exoskeleton(s)}

The ARMin exoskeleton is an upper-limb rehabilitation robot developed at the Sensory-Motor Systems Lab, ETH Zurich. The exoskeletal system has been conceived to promote task-oriented, repetitive, and intensive arm training in patients with upper extremities paralysis (Nef et al., 2009). In its early versions, ARMin is actuated with six DOFs. Four of them are controlled by implicit impedance control laws, while two are operated in admittance mode (Oldewurtel et al., 2007). The two DOFs controlled in admittance promote shoulder elevation and translation movements and rely on force/torque measurement of a 6-axis sensor at the shoulder joint. The robot is embedded with model-based friction and inverse dynamics compensation to improve transparency and minimize interaction forces with the human limb. In Nef et al. (2007), the ARMin II can operate in two different highlevel modalities, according to the patients' recovery stage. In passive mobilization mode, first, the therapist moves the patient's arm together with the robot on the desired trajectory. In this phase, the robot's gravity and friction are compensated so that the therapist feels only the forces and torques necessary to move the human arm. Then, an algorithm extracts relevant way-points during the movement and computes a minimum jerk trajectory to be followed by the passive mobilization therapy with adjustable velocity. The authors also implemented a ball game based on an assistive mode: the subject has to catch a virtual ball rolling down an inclined virtual table. Through a Cartesian-space impedance control law, the robot supports the user by driving their hand along the horizontal plane with gradient force-fields assistance that pushes the patient's arm towards the ball position. Guidali et al. (2011) presented a further development of ARMin III. The authors built a virtual tunnel around the task-space reference trajectory allowing the user to move freely within the tunnel while guided at the tunnel walls. Furthermore, the exercise could be triggered by the patients' voluntary activity when the user effort overcomes a certain threshold, and the movement is directed to the next task. In a recent study, the researchers improved the ARMin IV exoskeleton's transparency through a velocity-based disturbance observer. They compared it to the more traditional friction, gravity, and inertia feedforward compensation (Just et al., 2018). Finally, three distinct methods for arm weight compensation using the ARMin exoskeleton were proposed and analyzed (Just et al., 2020). All three methods are based on anthropometric arm models and are generalizable for use in different robotic devices and various subjects.

\subsection{The CleverARM Exoskeleton}

The CLEVERarm is an 8-DOFs lightweight and ergonomic upperlimb rehabilitation exoskeleton for upper-limb impairment developed at the Texas A\&M University, capable of producing diverse and perceptually rich training scenarios Soltani-Zarrin et al. (2017b); Zeiaee et al. (2019). The robot supports the motion of the shoulder girdle, glenohumeral joint, elbow, and wrist. Six degrees of freedom of the exoskeleton are active, and the two degrees of freedom supporting the wrist motion are passive. The CLEVERarm joints employ electric motors coupled with harmonic drive actuators, and the mechatronic system is provided with force/torque sensors at the two interaction points with the arm to enable achieving back-drivability of the exoskeleton. The control scheme relies on an impedance-based controller employed to track rehabilitation exercises implemented in the game environment. The path generator computes human-like motions that support the scapulohumeral rhythm Soltani-Zarrin et al. (2017). Additionally, the controller is provided with a gravitational model of the robot to cancel the weight of the exoskeleton, and a friction compensation method, achieved through an admittance-based controller. Finally, the interaction forces measured by the $\mathrm{F} / \mathrm{T}$ sensors are used to compute the desired velocity of the interaction ports to improve the back-drivability of the exoskeleton. Then, the desired angular velocities are computed through the system Jacobian and are fed as references to the impedance-based controller Soltani-Zarrin et al. (2018)

\subsection{The EXO-UL8 Exoskeleton}

The EXO-UL8 is a dual-arm exoskeleton that covers all the main movements of a human's upper limb. The robot supports the motion of the shoulder, elbow, and wrist through seven nonbackdrivable joints, and an additional joint operates the handgrip Shen et al. (2019). In a previous version, the EXO-UL7 was actuated through cable-driven actuation mechanisms. Now, the EXO-UL8 is operated by electric motors coupled with harmonic drives. A set of four force/torque sensors are placed at the physical interaction points between the user and the exoskeletal system. The robot relies on an admittance controller that allows the exoskeleton to behave transparently to user-driven movements. Precisely, torques applied by the human to the exoskeleton joints are estimated from the F/T sensors, then, through an admittance model, reference trajectories are generated and operated by the inner low-level PID control loops. Friction and gravity compensation is added as feedforward terms to the low-level controller Shen et al. (2019). The core concept of the EXO-UL8 controller is to generate motion in response to human-applied forces to improve backdrivability and reduce the user-perceived weight of the robot. On top of this, the system can be operated to follow pre-defined trajectories for rehabilitation exercises. Since the authors developed a dual-arm symmetric system, they also enabled mirroring training modes based on bilateral teleoperation between unimpaired and impaired arms. Additionally, Shen et al. (2018) proposed an asymmetric bilateral training using an interactive virtual reality environment.

\subsection{The FELXO-Arm1 Exoskeleton}

The FELXO-Arm1 system is an upper-limb exoskeleton for neurorehabilitation. It supports shoulder and elbow movements through 5-DOFs aligned with human upper-limb joints to match natural physiological synergies. Lin et al. (2021) presented a control strategy for customized robot-assisted passive rehabilitation. The method aims to coordinate shoulder and elbow movements during the early stage of the rehabilitation 
treatment. The authors proposed a teaching training strategy by which the therapist provides desired trajectories by driving the impaired limb of the patient in the workspace. The trajectory is then adjusted in position, velocity, and acceleration to promote movement smoothness and continuity. Then, the movement is repeated over time with high intensity. The exoskeleton joints are controlled by a proportional differential-based trajectory tracking controller based on an implicit impedance control law. The inverse dynamic model of the system is computed according to the Lagrange method, and the generated torques are included as a feedforward torque controller. The FELXO-Arm 1 exoskeleton is powered by BLDC electric motors coupled with harmonic drive gearboxes. A friction compensation algorithm rejects residual dynamic and breakthrough friction at each joint to improve transparency and compliant behavior. Torque sensors measure human-robot interactive torques at shoulder and elbow joints to identify the movement intention of the patient.

\subsection{The Harmony Exoskeleton}

Kim and Deshpande (2017) developed an upper-body bi-manual exoskeleton for post-stroke rehabilitation aimed at providing natural coordination at the shoulder complex. The robotic exoskeleton consists of seven degrees-of-freedom (DOFs) for each arm: five DOFs are used to assist the shoulder and the scapulohumeral rhythm, one DOFs assists the elbow flexion/extension, and one operates the wrist pronation/supination. The authors developed a baseline controller that implements active modalities with jointcoordination constraints (Kim and Deshpande, 2015; Dalla Gasperina et al., 2020). The baseline controller promotes joint transparency, corrects for non-coordinated scapulohumeral rhythm through an impedance control law (spring-damper corrective assistance), and compensates for the robot weight through positive feedback that is computed inverse dynamics recursive algorithm (weight counterbalance assistance). The exoskeleton is actuated with SEAs, which are used to compute an indirect measure of torque by measuring the deformation of the elastic element. The exoskeleton can also be operated with an explicit joint-space impedance control scheme to follow desired trajectories Oliveira et al. (2019), and it can promote different high-level modalities ranging from assistive to resistive modalities. Finally, the mechatronic system is also provided with force/torque 6-axis sensors at the interaction upper arm and forearm cuffs, and it can be operated to display a desired stiffness in the Cartesian space as well (Kim and Deshpande, 2017).

\subsection{The L-EXOS Exoskeleton}

Frisoli et al. (2005) developed a force-feedback cable-driven light exoskeleton (L-EXOS) for rehabilitation. The L-EXOS robot operates four active DOFs: three DOFs assist the shoulder ball-socket joint, and one DOF is devoted to the elbow movements. In Frisoli et al. (2009), the robot is controlled in assistive mode, and the robot assists the motion only when the subject is not able to complete the rehabilitation exercise. In detail, the controller discriminates longitudinal and orthogonal direction with respect to the reference trajectory and promotes a virtual tunnel that follows the task-space desired trajectory. From a lower-level perspective, two concurrent task-space impedance controllers act along the tangential and orthogonal directions of the trajectory and compute the desired restoring forces at the end-effector. The transposed Jacobian matrix is used to convert task-space forces to joint-space torques, that are actuated accordingly. The dynamic model of the exoskeleton is derived from CAD models and the weight compensation of the device is implemented with a feedforward torque contribution. The robot is actuated with permanent magnet torque actuators, that do provide intrinsic backdrivability. Also, the design of the exoskeleton was conducted following a set of guidelines to improve the transparency of the device, such as choosing high power density actuators, low transmission ratios, and low backlash gearboxes. Furthermore, motors were placed remotely with respect to the actuation joint using tendon transmissions to minimize the perceived inertia due to the motors' weight.

\subsection{The NEUROExos Shoulder-Elbow Module Exoskeleton}

The NEUROExos Shoulder-Elbow Module (NESM) is an exoskeleton for upper-limb neurorehabilitation and spasticity treatment. It actuates 4-DOFs, namely 3-DOFs at the shoulder and 1-DOF at the elbow (Crea et al., 2016). The exoskeleton joints are composed of high-torque SEA units, which permit highfidelity torque control and introduce mechanical compliance to accommodate users' voluntary movements. Torque-sensing is achieved in joint-space by indirect measurement on the seriesspring displacement. The robot can operate in various training modalities, such as passive mobilization, active-assisted, activeresisted, and active-disturbed training modes. In order to adapt the robot assistance to a wide range of patients' residual movements, the exoskeleton is provided with two control macromodalities: robot-in-charge and patient-in-charge programs (Trigili et al., 2019). In the robot-in-charge approach, the robot passively mobilizes the human arm along pre-defined joint trajectories. The joints are position-controlled through a standard PID scheme, and the intrinsic serial elasticity provides additional compliance to accommodate spasticity and uncomfortable positions. Join trajectories are computed through an inverse-kinematics algorithm by selecting maximum joint or hand velocities. Instead, in patient-incharge mode, each joint of the exoskeleton is torque-controlled. In particular, the feedback torque is derived from the series-spring elongation, and a PID scheme tracks the desired torque. In this macro-mode, the robot promotes compliant control, behaves transparently to user-initiated movements, assists and resists the user's movements at each joint according to the desired training modality. The authors also present some additional sub-modes. In transparent mode, the robot tracks the null-torque and compensates for its weight. In impedance control mode, a dual (convergent and divergent) explicit impedance control scheme assists (or disturbs) the motion along the desired joint trajectories. Finally, three variants of muscle strength training modes are implemented to training specific muscular groups in a resistive-like manner. In all patient-in-charge sub-modalities, a gravity compensation algorithm iteratively computes the gravity torque of each joint due to the robot weight. Then, the gravity torque is fed as a torque feedforward contribution to the central controller (Crea et al., 2017). 
TABLE 3 | Comparison of available control strategies at high-level, low-level and hardware-level for upper-limb neurorehabilitation exoskeletons. S: shoulder, E: elbow, W: wrist.

\begin{tabular}{|c|c|c|c|c|c|c|c|c|}
\hline Exoskeleton & $\begin{array}{l}\text { Supported } \\
\text { joints }\end{array}$ & DOFs & Bimanual & High-level & Low-level & Actuation & Sensing & References \\
\hline AAU & $S, E$ & 3 & no & None & $\begin{array}{l}\text { Admittance (with gravity } \\
\text { compensation) }\end{array}$ & $\begin{array}{l}\text { Brushless motor } \\
\text { coupled with } \\
\text { harmonic drive } \\
\text { gearbox }\end{array}$ & $\begin{array}{l}\text { Task-space } \\
\text { force sensing } \\
\text { resistors (FSR) }\end{array}$ & Bai et al. (2017) \\
\hline ALEx & S,E & $4 \times 2$ & yes & $\begin{array}{l}\text { Passive (passive- } \\
\text { triggered), Active- } \\
\text { assistive (adaptive } \\
\text { assistance) and Active } \\
\text { modalities }\end{array}$ & $\begin{array}{l}\text { Position control of } \\
\text { compliant joints, Task- } \\
\text { space force control, } \\
\text { Cartesian impedance } \\
\text { control (with friction, } \\
\text { gravity and inertia } \\
\text { compensation) }\end{array}$ & $\begin{array}{l}\text { Brushless DC motor } \\
\text { coupled with } \\
\text { mechanically } \\
\text { compliant cable } \\
\text { transmission }\end{array}$ & no & $\begin{array}{l}\text { Pirondini et al. } \\
\text { (2016), Stroppa } \\
\text { et al. (2017b) }\end{array}$ \\
\hline ARAMIS & $\mathrm{S}, \mathrm{E}, \mathrm{W}$ & $6 \times 2$ & yes & $\begin{array}{l}\text { Passive (mirroring), } \\
\text { Active-assistive } \\
\text { (counterbalance) }\end{array}$ & $\begin{array}{l}\text { Position control of SEA- } \\
\text { based joints }\end{array}$ & $\begin{array}{l}\text { SEA-based brushed } \\
\text { DC motor coupled } \\
\text { with gearbox }\end{array}$ & $\begin{array}{l}\text { Joint-space } \\
\text { (SEA-based } \\
\text { indirect) }\end{array}$ & $\begin{array}{l}\text { Colizzi et al. } \\
\text { (2009), Pignolo } \\
\text { et al. (2012, } \\
\text { 2016) }\end{array}$ \\
\hline ARMin & $\mathrm{S}, \mathrm{E}, \mathrm{W}$ & 6 & no & $\begin{array}{l}\text { Passive, Active-assistive } \\
\text { (corrective, tunneling) }\end{array}$ & $\begin{array}{l}\text { Joint-space and task- } \\
\text { space implicit impedance } \\
\text { control (with friction and } \\
\text { dynamics compensation) }\end{array}$ & $\begin{array}{l}\text { Brushed DC motor } \\
\text { coupled with } \\
\text { harmonic drive } \\
\text { gearbox }\end{array}$ & $\begin{array}{l}\text { Task-space } \\
\text { (6-axis F/T } \\
\text { sensor, only } \\
\text { ARMin II } \\
\text { and III) }\end{array}$ & $\begin{array}{l}\text { Nef et al. (2009), } \\
\text { Guidali et al. } \\
\text { (2011), Just et al. } \\
\text { (2020) }\end{array}$ \\
\hline CLEVERarm & S,E,W & 6 & no & $\begin{array}{l}\text { Passive, and Active- } \\
\text { assistive }\end{array}$ & $\begin{array}{l}\text { Joint-space impedance } \\
\text { control (with friction and } \\
\text { gravity compensation) } \\
\text { and admittance-based } \\
\text { control for back-drivability }\end{array}$ & $\begin{array}{l}\text { Electric DC motor } \\
\text { coupled with } \\
\text { harmonic drive } \\
\text { gearboxes }\end{array}$ & $\begin{array}{l}\text { Task-space } \\
\text { (6-axis F/T } \\
\text { sensors) }\end{array}$ & $\begin{array}{l}\text { Soltani-Zarrin } \\
\text { et al. (2017b) }\end{array}$ \\
\hline EXO-UL8 & S,E,W & 7 & yes & $\begin{array}{l}\text { Passive and Active- } \\
\text { assistive (symmetric and } \\
\text { asymmetric mirroring), } \\
\text { Active (transparent) } \\
\text { modalities }\end{array}$ & $\begin{array}{l}\text { Task-space admittance } \\
\text { control (with friction and } \\
\text { gravity compensation) } \\
\text { and inner joint-space } \\
\text { position control }\end{array}$ & $\begin{array}{l}\text { Electric DC motor } \\
\text { coupled with } \\
\text { harmonic drive } \\
\text { gearboxes }\end{array}$ & $\begin{array}{l}\text { Task-space } \\
\text { (6-axis F/T } \\
\text { sensors) }\end{array}$ & $\begin{array}{l}\text { Shen et al. } \\
(2018,2019)\end{array}$ \\
\hline $\begin{array}{l}\text { FELXO- } \\
\text { Arm1 }\end{array}$ & $S, E$ & 5 & no & $\begin{array}{l}\text { Passive (teach-and- } \\
\text { replay), Active-assistive }\end{array}$ & $\begin{array}{l}\text { joint-space implicit } \\
\text { impedance control }\end{array}$ & $\begin{array}{l}\text { Brushless DC motor } \\
\text { coupled with } \\
\text { harmonic drive } \\
\text { gearboxes }\end{array}$ & $\begin{array}{l}\text { Joint-space } \\
\text { (direct) }\end{array}$ & Lin et al. (2021) \\
\hline Harmony & $\mathrm{S}, \mathrm{E}, \mathrm{W}$ & $7 \times 2$ & yes & $\begin{array}{l}\text { Active-assistive } \\
\text { (corrective and } \\
\text { counterbalance), Active } \\
\text { (inter-joint coordination) } \\
\text { and Resistive modalities }\end{array}$ & $\begin{array}{l}\text { Explicit impedance } \\
\text { control (with friction and } \\
\text { dynamics compensation) }\end{array}$ & $\begin{array}{l}\text { SEA-based } \\
\text { brushless DC motor }\end{array}$ & $\begin{array}{l}\text { Joint-space } \\
\text { (SEA-based } \\
\text { indirect), task- } \\
\text { space (6-axis } \\
\text { F/T sensors) }\end{array}$ & $\begin{array}{l}\text { Kim and } \\
\text { Deshpande } \\
\text { (2017) }\end{array}$ \\
\hline L-Exos & S,E & 4 & no & $\begin{array}{l}\text { Active-assistive } \\
\text { (corrective), Active } \\
\text { (tunneling) }\end{array}$ & $\begin{array}{l}\text { Task-space implicit } \\
\text { impedance control (with } \\
\text { friction and gravity } \\
\text { compensation) }\end{array}$ & $\begin{array}{l}\text { Quasi-backdrivable } \\
\text { permanent magnet } \\
\text { torque motor }\end{array}$ & no & $\begin{array}{l}\text { Frisoli et al. } \\
(2005,2009)\end{array}$ \\
\hline NESM & S,E & 4 & no & $\begin{array}{l}\text { Passive, Active-assistive, } \\
\text { Resistive (viscous-field } \\
\text { and error-augmentation) }\end{array}$ & $\begin{array}{l}\text { Position control of SEA- } \\
\text { based joints, joint-space } \\
\text { explicit impedance } \\
\text { control }\end{array}$ & $\begin{array}{l}\text { SEA-based } \\
\text { brushless DC motor } \\
\text { coupled with } \\
\text { harmonic drive } \\
\text { gearboxes and } \\
\text { custom springs }\end{array}$ & $\begin{array}{l}\text { Joint-space } \\
\text { (SEA-based } \\
\text { indirect) }\end{array}$ & $\begin{array}{l}\text { Crea et al. } \\
\text { (2016); Trigili } \\
\text { et al. (2019) }\end{array}$ \\
\hline NTUH-II & S,E,W & 8 & no & $\begin{array}{l}\text { Active-assistive (velocity- } \\
\text { field control) }\end{array}$ & Admittance control & $\begin{array}{l}\text { Brushless DC motor } \\
\text { coupled with } \\
\text { gearbox }\end{array}$ & $\begin{array}{l}\text { Task-space } \\
\text { (6-axis } F / T \\
\text { sensors at } \\
\text { wrist and } \\
\text { upper arm) }\end{array}$ & $\begin{array}{l}\text { Lin et al. (2014); } \\
\text { Chia et al. (2020) }\end{array}$ \\
\hline Pneu-WREX & S,E & 4 & no & $\begin{array}{l}\text { Active-assistive } \\
\text { (assistance-as-needed) }\end{array}$ & $\begin{array}{l}\text { Model-based adaptive } \\
\text { impedance control }\end{array}$ & Pneumatic actuation & no & $\begin{array}{l}\text { Wolbrecht et al. } \\
\text { (2008) }\end{array}$ \\
\hline RECUPERA & S,E,W & $5 \times 2$ & yes & $\begin{array}{l}\text { Passive (mirroring), } \\
\text { Active-assistive } \\
\text { (counterbalance) }\end{array}$ & $\begin{array}{l}\text { Position, velocity, current } \\
\text { control }\end{array}$ & $\begin{array}{l}\text { Brushless DC motor } \\
\text { coupled with low- } \\
\text { backlash gearboxes }\end{array}$ & $\begin{array}{l}\text { task-space }(6- \\
\text { axis F/T } \\
\text { sensors) }\end{array}$ & $\begin{array}{l}\text { Kumar et al. } \\
\text { (2019) }\end{array}$ \\
\hline
\end{tabular}




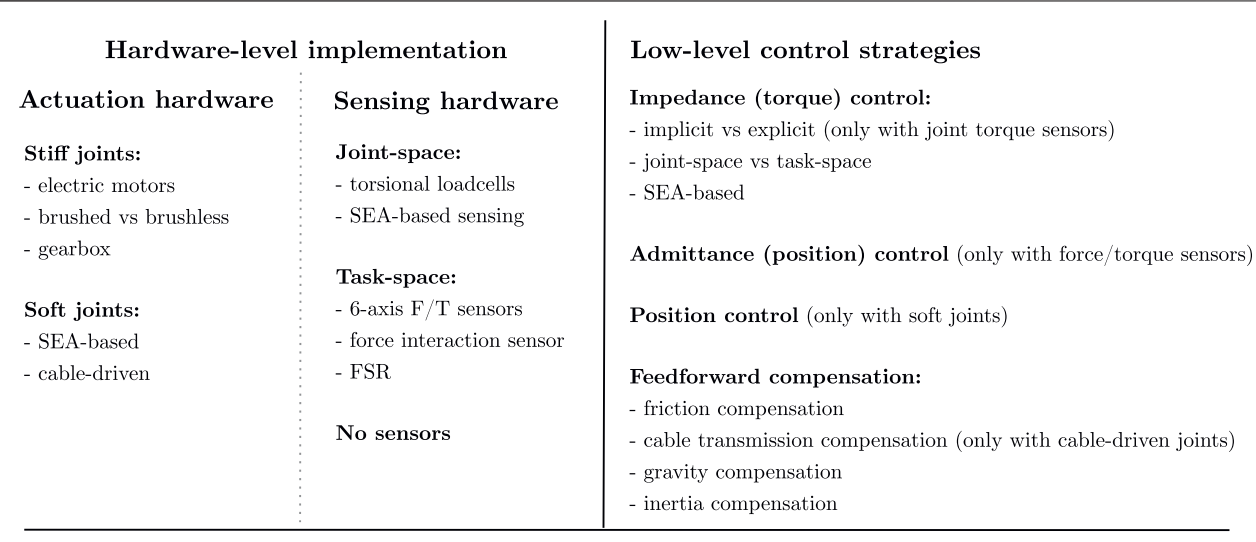

High-level training modalities

Passive:
- passive mobilization
- passive triggered
- teach-and-replay
- mirror training

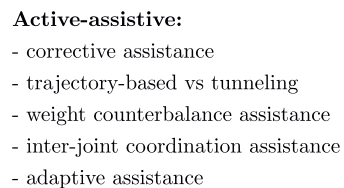

\section{Resistive:}

- viscous-field resistance

- ROM boundaries

- tunneling strategies

trajectory-constrained

strategies

FIGURE 8 | Summary of patient-cooperative control strategies for upper-limb rehabilitation exoskeletons at different levels of implementation: hardware-level actuation and sensing implementation, low-level control strategies, and high-level training modalities.

\subsection{The NTUH-II Exoskeleton}

The NTUH-II exoskeleton is an upper-limb device for robotic rehabilitation for shoulder-impaired patients (Lin et al., 2014). Such exoskeleton is provided with 8 DOFs, being able to reproduce most of the shoulder movements, such as shoulder flexion/extension, horizontal abduction/adduction, and rotation. The exoskeleton has been provided with the following control schema (Chia et al., 2020). A Kalman filter has been designed in order to estimate the human torques. An admittance model is then used to access the active motion of the human (therefore, making it possible to estimate the user's intention of motion). On top of that, a velocity field is designed in order to provide active assistance to the subject in interaction with the exoskeleton. Finally, an integration method is proposed in order to combine the admittance model output with the velocity field output, providing the reference signal to the exoskeleton controller, computing the torques to be applied by the motors. The main contribution that has been given by the proposed control approach is related to the velocity field: it provides a method for the generation of time-independent assistance based on the given rehabilitation task. In order to implement the proposed velocity field, the considered rehabilitation task has to be parameterized. After that, the path is encoded using the velocity field to make the assistance time-independent. The adopted velocity field is capable to assist the subject to execute the target task along the tangential direction of the reference trajectory while compensating for deviations along the normal directions. Experimental results have demonstrated the capabilities of the proposed approach to assist the subject during the rehabilitation task execution.

\subsection{The Pneu-WREX Exoskeleton}

The Pneu-WREX exoskeleton is an upper-limb device for robotaided movement training following stroke (Wolbrecht et al.,
2008). The Pneu-WREX exoskeleton is provided with 4 pneumatically actuated DOFs. The following three main characteristics have been implemented in the proposed device: mechanical compliance, the ability to assist patients in completing desired movements, and the ability to provide only the minimum necessary assistance. In order to provide active assistance to the subject, the exoskeleton is controlled exploiting two control loops: an inner controller based on a standard modelbased, adaptive control approach in order to learn the patient's abilities and assist in completing movements while remaining compliant, and an outer assistance-as-needed controller defining a force term to the adaptive control law. Such an outer controller decays the force output from the robot when errors in task execution are small, while it increases the assistance to the user when errors in task execution are bigger. The proposed controller has been demonstrated to be successful in experimental tests executed with people who have suffered a stroke.

\subsection{The RECUPERA Exoskeleton}

Kumar et al. (2019) recently presented a lightweight dual-arm rehabilitation robot called RECUPERA exoskeleton. The exoskeleton offers a high level of modularity. It can be used as a wheelchair-mounted system or as a full-body system for therapistguided and self-training for neurorehabilitation of the upper body. The wheelchair-mounted version features 5-DOFs for each arm, while the full-body operates 30 DOFs. The RECUPERA exoskeleton implements three different rehabilitation training modalities, namely gravity compensation, teach-and-replay, and master-slave therapy. In gravity compensation mode, the robot compensates for its weight through an inverse dynamic model of the exoskeleton. This mode can also include the compensation of the human arms dynamic model, and it is conceived as the baseline 
controller of the robot. The teach-and-replay mode consists of two phases. First, the robot is operated in gravity compensation mode, and the therapist performs a trajectory that is recorded by the system. Afterward, the robot detects a trigger from the user (or from the therapist) and passively performs the recorded movement. Finally, the master-slave mode consists of a mirroring strategy by which the paretic arm follows and mimics the trajectory performed with the healthy arm operated in gravity compensation mode. The RECUPERA exoskeleton is powered by high-torque BLDC actuators coupled with low-backlash gearboxes to increase torque output at the joint axis. The joints are controlled with cascaded position, velocity, and current control loops, while torque control is achieved through motor current measurements. The exoskeleton is also provided with 6-DOFs force/torque sensors to detect human-robot interaction at the three interfaces: hand, forearm, and upper arm interaction ports.

\section{DISCUSSION AND PERSPECTIVE}

Control advancements for upper-limb exoskeletons for rehabilitation are spreading rapidly, and the literature continuously presents new prototypes and control approaches. Since we noticed that state-of-the-art reviews on controls for rehabilitation robots generally focus their attention on training modalities and human-robot interaction, our study was intended to propose a multi-disciplinary taxonomy of patient-cooperative control strategies for rehabilitation upper-limb exoskeletons that could help researchers develop complex and advanced systems. Our classification is based on a three-level scheme: on 1) highlevel training modalities, 2) low-level control strategies, and 3) hardware-level implementation. Overall, we report that most high-level modalities are based on assistive approaches, by which the robot partially supports the user during the motion. In turn, most exoskeletons support human movements in three ways. On one side, they provide corrective assistance, either through impedance-based strategies or via tunneling methods that guide the user to stay within a specific virtual path. Alternatively, weight counterbalance, also known as antigravity support, can be implemented to compensate for gravity due to the user's arm. Finally, inter-joint coordination is involved whether the aim is to induce physiological coordination based on position, torque, or velocity-based synergies.

As shown in Table 3, different "low-level" control strategies can be used to promote the same "high-level" modalities, and there is not a unique relationship between the three layers. The majority of upperlimb exoskeletons are conceived upon the foundation of compliant control, by which the user should have the lead when performing the rehabilitation task. The robot should always follow the user's intention and apply corrective actions only when the residual muscular forces are insufficient to fulfill the action. Among low-level compliant control strategies, impedance control is the most used since it outperforms admittance control strategies when low-impedance behavior (i.e., transparent free motion) is desired. Furthermore, we underline the importance of achieving high-quality compliant control, either through high-fidelity torque control in conjunction with impedance control strategies or through torque/force-sensing combined with admittance control. Indeed, many researchers rely on force feedback, which can be obtained either via SEA-based indirect measurements or torsional/linear loadcell-based direct measurements. Many studies demonstrated that torque feedback could improve the performances of compliant control over modelbased compensation methods. However, the introduction of additional sensors can drastically increase prototype costs. Series elastic actuators are gaining increasing interest since they provide inexpensive, accurate torque sensing at the joint and introduce mechanical compliance to promote compliant motion. Furthermore, with SEAs, it is impossible to achieve higher stiffness than the elastic element, reducing the robot performances when highimpedance (rigid) interaction is required.

In Figure 8, we report a summary of the aspects we investigated in this work, intending to help robotics researchers bridge the gap between desired rehabilitation outcomes and robotic implementation. The reader can interpret the scheme following both bottom-up and top-down paradigms: 1) the exoskeleton control design process can both start from the available hardware (actuator, sensors, etc.) to define the implementable control strategies for the desired behavior, or 2) researchers can identify the hardware requirements from the selected high-level training modalities.

In conclusion, this review presents an interdisciplinary vision on control solutions for upper-limb exoskeletons that suggest how different approaches can render physical human-robot interaction at different levels of implementation to promote the desired rehabilitation behavior. We noticed that most high-level training modalities are derived from motor learning concepts to improve the rehabilitation outcomes, and exoskeletons are usually programmed to mimic the therapist's actions during conventional treatment. We suggest that to exploit robotic assistance to promote motor recovery, more neurologicalinspired modalities should be investigated to deepen the effects of robot-mediated therapy on neural plasticity and motor relearning. Finally, further research is needed to evaluate which approach (at each level) is associated with a more significant improvement of arm functions after stroke.

\section{AUTHOR CONTRIBUTIONS}

SDG, LR, AP, FB, MG conceived the study and the structure of the review. SDG developed the theory and organized the preliminary manuscript, tables, and figures. SDG, MG, and LR drafted the contents and the manuscript. All authors read, reviewed, and approved the final manuscript prior to submission.

\section{FUNDING}

We gratefully acknowledge the funding provided by the EMPATIA@LECCO project (Cariplo Foundation and Regione Lombardia, Erogazioni Emblematiche Maggiori 2016, Ref. 20161428) and the AGREE project (Regione Lombardia, Precommercial procurement, Ref. ARCA_2018_132). 


\section{REFERENCES}

Abdollahi, F., Case Lazarro, E. D., Listenberger, M., Kenyon, R. V., Kovic, M., Bogey, R. A., et al. (2014). Error Augmentation Enhancing Arm Recovery in Individuals With Chronic Stroke. Neurorehabil. Neural Repair. 28, 120-128. doi:10.1177/1545968313498649

Ambrosini, E., Ferrante, S., Zajc, J., Bulgheroni, M., Baccinelli, W., d'Amico, E., et al. (2017). The Combined Action of a Passive Exoskeleton and an EMGControlled Neuroprosthesis for Upper Limb Stroke Rehabilitation: First Results of the RETRAINER Project. IEEE Int. Conf. Rehabil. Robotics 56-61. doi:10.1109/ICORR.2017.8009221

Ambrosini, E., Gasperini, G., Zajc, J., Immick, N., Augsten, A., Rossini, M., et al. (2021). A Robotic System With EMG-Triggered Functional Eletrical Stimulation for Restoring Arm Functions in Stroke Survivors. Neurorehabil. Neural Repair. 35, 334-345. doi:10.1177/1545968321997769

Armstrong, B. (1988). "Friction: Experimental Determination, Modeling and Compensation," in Proceedings. 1988 IEEE International Conference on Robotics and Automatio (IEEE Comput. Soc. Press), 1422-1427. doi:10.1109/ROBOT.1988.12266n

Bai, S., Christensen, S., and Islam, M. R. U. (2017). "An Upper-Body Exoskeleton With a Novel Shoulder Mechanism for Assistive Applications," in IEEE/ASME International Conference on Advanced Intelligent Mechatronics, AIM, Munich, Germany (Institute of Electrical and Electronics Engineers Inc), 1041-1046. doi:10.1109/AIM.2017.8014156

Ballester, B. R., Maier, M., Duff, A., Cameirão, M., Bermúdez, S., Duarte, E., et al. (2019). A Critical Time Window for Recovery Extends Beyond One-Year PostStroke. J. Neurophysiol. 122, 350-357. doi:10.1152/jn.00762.2018

Barsotti, M., Leonardis, D., Loconsole, C., Solazzi, M., Sotgiu, E., Procopio, C., et al. (2015). "A Full Upper Limb Robotic Exoskeleton for Reaching and Grasping Rehabilitation Triggered by MI-BCI," in 2015 IEEE International Conference on Rehabilitation Robotics (ICORR) (IEEE), 49-54. doi:10.1109/ ICORR.2015.7281174

Basteris, A., Nijenhuis, S. M., Stienen, A. H., Buurke, J. H., Prange, G. B., and Amirabdollahian, F. (2014). Training Modalities in Robot-Mediated Upper Limb Rehabilitation in Stroke: A Framework for Classification Based on a Systematic Review. J. NeuroEngineering Rehabil. 11, 111-115. doi:10.1186/ 1743-0003-11-111

Bastian, A. J. (2008). Understanding Sensorimotor Adaptation and Learning for Rehabilitation. Curr. Opin. Neurol. 21, 628-633. doi:10.1097/ WCO.0b013e328315a293

Bernhardt, J., Hayward, K. S., Kwakkel, G., Ward, N. S., Wolf, S. L., Borschmann, K., et al. (2017). Agreed Definitions and a Shared Vision for New Standards in Stroke Recovery Research: The Stroke Recovery and Rehabilitation Roundtable Taskforce. Int. J. Stroke 12, 444-450. doi: $10.1177 / 1747493017711816$

Bertani, R., Melegari, C., De Cola, M. C., Bramanti, A., Bramanti, P., and Calabrò, R. S. (2017). Effects of Robot-Assisted Upper Limb Rehabilitation in Stroke Patients: a Systematic Review With Meta-Analysis. Neurol. Sci. 38, 1561-1569. doi:10.1007/s10072-017-2995-5

Bigoni, M., Baudo, S., Cimolin, V., Cau, N., Galli, M., Pianta, L., et al. (2016). Does Kinematics Add Meaningful Information to Clinical Assessment in Post-Stroke Upper Limb Rehabilitation? A Case Report. J. Phys. Ther. Sci. 28, 2408-2413. doi:10.1589/jpts.28.2408

Boaventura, T., Medrano-Cerda, G. A., Semini, C., Buchli, J., and Caldwell, D. G. (2013). "Stability and Performance of the Compliance Controller of the Quadruped Robot HyQ," in IEEE International Conference on Intelligent Robots and Systems, 1458-1464. doi:10.1109/IROS.2013.6696541

Boaventura, T., Semini, C., Buchli, J., Frigerio, M., Focchi, M., and Caldwell, D. G. (2012). "Dynamic Torque Control of a Hydraulic Quadruped Robot," in Proceedings - IEEE International Conference on Robotics and Automation, 1889-1894. doi:10.1109/ICRA.2012.6224628

Brackenridge, J., V. Bradnam, L., Lennon, S., J. Costi, J., and A. Hobbs, D. (2016). A Review of Rehabilitation Devices to Promote Upper Limb Function Following Stroke. Neurosci. Biomed. Eng. 4, 25-42. doi:10.2174/ 2213385204666160303220102

Brauchle, D., Vukelić, M., Bauer, R., and Gharabaghi, A. (2015). Brain StateDependent Robotic Reaching Movement With a Multi-Joint Arm Exoskeleton:
Combining Brain-Machine Interfacing and Robotic Rehabilitation. Front. Hum. Neurosci. 9, 564. doi:10.3389/fnhum.2015.00564

Brokaw, E. B., Holley, R. J., and Lum, P. S. (2013). Comparison of Joint Space and End Point Space Robotic Training Modalities for Rehabilitation of Interjoint Coordination in Individuals With Moderate to Severe Impairment From Chronic Stroke. IEEE Trans. Neural Syst. Rehabil. Eng. 21, 787-795. doi:10.1109/TNSRE.2013.2238251

Calanca, A., Muradore, R., and Fiorini, P. (2016). A Review of Algorithms for Compliant Control of Stiff and Fixed-Compliance Robots. IEEE/ASME Trans. Mechatron. 21, 613-624. doi:10.1109/TMECH.2015.2465849

Calanca, A., Muradore, R., and Fiorini, P. (2017). Impedance Control of Series Elastic Actuators: Passivity and Acceleration-Based Control. Mechatronics 47, 37-48. doi:10.1016/j.mechatronics.2017.08.010

Chang, J.-J., Tung, W.-L., Wu, W.-L., Huang, M.-H., and Su, F.-C. (2007). Effects of Robot-Aided Bilateral Force-Induced Isokinetic Arm Training Combined With Conventional Rehabilitation on Arm Motor Function in Patients With Chronic Stroke. Arch. Phys. Med. Rehabil. 88, 1332-1338. doi:10.1016/ j.apmr.2007.07.016

Chen, T., Casas, R., and Lum, P. S. (2019). An Elbow Exoskeleton for Upper Limb Rehabilitation With Series Elastic Actuator and Cable-Driven Differential. IEEE Trans. Robot. 35, 1464-1474. doi:10.1109/TRO.2019.2930915

Chia, E.-Y., Chen, Y.-L., Chien, T.-C., Chiang, M.-L., Fu, L.-C., Lai, J.-S., et al. (2020). "Velocity Field Based Active-Assistive Control for Upper Limb Rehabilitation Exoskeleton Robot," in 2020 IEEE International Conference on Robotics and Automation (ICRA) (IEEE), 1742-1748. doi:10.1109/ ICRA40945.2020.9196766

Christensen, S., and Bai, S. (2018). Kinematic Analysis and Design of a Novel Shoulder Exoskeleton Using a Double Parallelogram Linkage. J. Mech. Robotics 10. doi:10.1115/1.4040132

Cieza, A., Causey, K., Kamenov, K., Hanson, S. W., Chatterji, S., and Vos, T. (2020). Global Estimates of the Need for Rehabilitation Based on the Global Burden of Disease Study 2019: a Systematic Analysis for the Global Burden of Disease Study 2019. The Lancet 396, 2006-2017. doi:10.1016/S0140-6736(20)32340-0

Colizzi, L., Lidonnici, A., and Pignolo, L. (2009). The ARAMIS Project: A Concept Robot and Technical Design. J. Rehabil. Med. 41, 1011-1101. doi:10.2340/ 16501977-0407

Colombo, R., Pisano, F., Micera, S., Mazzone, A., Delconte, C., Carrozza, M. C., et al. (2005). Robotic Techniques for Upper Limb Evaluation and Rehabilitation of Stroke Patients. IEEE Trans. Neural Syst. Rehabil. Eng. 13, 311-324. doi:10.1109/TNSRE.2005.848352

Crea, S., Cempini, M., Moise, M., Baldoni, A., Trigili, E., Marconi, D., et al. (2016). "A Novel Shoulder-Elbow Exoskeleton With Series Elastic Actuators," in Proceedings of the IEEE RAS and EMBS International Conference on Biomedical Robotics and Biomechatronics, 1248-1253. doi:10.1109/ BIOROB.2016.7523802

Crea, S., Cempini, M., Moisè, M., Baldoni, A., Trigili, E., Marconi, D., et al. (2017). Validation of a Gravity Compensation Algorithm for a Shoulder-Elbow Exoskeleton for Neurological Rehabilitation. Biosyst. Biorobotics. 15, 495-499. doi:10.1007/978-3-319-46669-9 82

Crespo, L. M., and Reinkensmeyer, D. J. (2008). "Effect of Robotic Guidance on Motor Learning of a Timing Task," in Proceedings of the 2nd Biennial IEEE/ RAS-EMBS International Conference on Biomedical Robotics and Biomechatronics, BioRob, 199-204. doi:10.1109/BIOROB.2008.4762796

Crocher, V., Sahbani, A., and Morel, G. (2010). "mposing Joint Kinematic Constraints With an Upper Limb Exoskeleton Without Constraining the End-Point Motion," in IEEE/RSJ 2010 International Conference on Intelligent Robots and Systems, IROS 2010 - Conference Proceedings, 5028-5033. doi:10.1109/IROS.2010.5650961

De Oliveira, A. C., Sulzer, J. S., and Deshpande, A. D. (2021). Assessment of UpperExtremity Joint Angles Using Harmony Exoskeleton. IEEE Trans. Neural Syst. Rehabil. Eng. 29, 916-925. doi:10.1109/TNSRE.2021.3074101

Dipietro, L., Ferraro, M., Palazzolo, J. J., Krebs, H. I., Volpe, B. T., and Hogan, N. (2005). Customized Interactive Robotic Treatment for Stroke: EMG-Triggered Therapy. IEEE Trans. Neural Syst. Rehabil. Eng. 13, 325-334. doi:10.1109/ TNSRE.2005.850423

Dolce, G., Lucca, L., and Pignolo, L. (2009). Robot-assisted Rehabilitation of the Paretic Upper Limb: Rationale of the ARAMIS Project. J. Rehabil. Med. 41, 1007-1101. doi:10.2340/16501977-0406 
Emken, J. L., Benitez, R., and Reinkensmeyer, D. J. (2007a). Human-Robot Cooperative Movement Training: Learning a Novel Sensory Motor Transformation during Walking with Robotic Assistance-As-Needed. J. Neuroengineering Rehabil. 4, 1-16. doi:10.1186/1743-0003-4-8

Emken, J. L., Benitez, R., Sideris, A., Bobrow, J. E., and Reinkensmeyer, D. J. (2007b). Motor Adaptation as a Greedy Optimization of Error and Effort. J. Neurophysiol. 97, 3997-4006. doi:10.1152/jn.01095.2006

Focchi, M., Medrano-Cerda, G. A., Boaventura, T., Frigerio, M., Semini, C., Buchli, J., et al. (2016). Robot Impedance Control and Passivity Analysis With Inner Torque and Velocity Feedback Loops. Control Theor. Technol. 14, 97-112. doi:10.1007/s11768-016-5015-z

Frisoli, A., Loconsole, C., Leonardis, D., Banno, F., Barsotti, M., Chisari, C., et al. (2012). A New Gaze-BCI-Driven Control of an Upper Limb Exoskeleton for Rehabilitation in Real-World Tasks. IEEE Trans. Syst. Man. Cybern. C. 42, 1169-1179. doi:10.1109/TSMCC.2012.2226444

Frisoli, A., Rocchi, F., Marcheschi, S., Dettori, A., Salsedo, F., and Bergamasco, M. (2005). "A New Force-Feedback Arm Exoskeleton for Haptic Interaction in Virtual Environments," in Proceedings - 1st Joint Eurohaptics Conference and Symposium on Haptic Interfaces for Virtual Environment and Teleoperator Systems; World Haptics Conference, WHC 2005, Pisa, Italy (Institute of Electrical and Electronics Engineers Inc), 195-201. doi:10.1109/WHC.2005.15

Frisoli, A., Salsedo, F., Bergamasco, M., Rossi, B., and Carboncini, M. C. (2009). A Force-Feedback Exoskeleton for Upper-Limb Rehabilitation in Virtual Reality. Appl. Bionics Biomech. 6, 115-126. doi:10.1080/11762320902959250

Gandolla, M., Ferrante, S., Baldassini, D., Cotti Cottini, M., Seneci, C., Molteni, F., et al. (2016a). EMG-Controlled Robotic Hand Rehabilitation Device for Domestic Training. IFMBE Proc. 57, 638-642. doi:10.1007/978-3-319-327037-12410.1007/978-3-319-32703-7_124

Gandolla, M., Ward, N. S., Molteni, F., Guanziroli, E., Ferrigno, G., and Pedrocchi, A. (2016b). The Neural Correlates of Long-Term Carryover Following Functional Electrical Stimulation for Stroke. Neural Plasticity 2016, 1-13. doi:10.1155/2016/4192718

Gandolla, M., Ferrante, S., Molteni, F., Guanziroli, E., Frattini, T., Martegani, A., et al. (2014). Re-thinking the Role of Motor Cortex: Context-Sensitive Motor Outputs? NeuroImage. 91, 366-374. doi:10.1016/j.neuroimage.2014.01.011

Gandolla, M., Niero, L., Molteni, F., Guanziroli, E., Ward, N. S., and Pedrocchi, A. (2021). Brain Plasticity Mechanisms Underlying Motor Control Reorganization: Pilot Longitudinal Study on Post-Stroke Subjects. Brain Sci. 11, 329. doi:10.3390/BRAINSCI11030329

Dalla Gasperina, S., Ghonasgi, K., De Oliveira, A. C., Gandolla, M., Pedrocchi, A., Deshpande, A., et al. (2020). "A Novel Inverse Kinematics Method for UpperLimb Exoskeleton under Joint Coordination Constraints," in IEEE International Conference on Intelligent Robots and Systems, Las Vegas, NV, USA (Institute of Electrical and Electronics Engineers Inc), 3404-3409. doi:10.1109/IROS45743.2020.9341686

Ghonasgi, K., Rose, C. G., De Oliveira, A. C., Varghese, R. H., and Deshpande, A. D. (2021). "Design and Validation of a Novel Exoskeleton Hand Interface: The Eminence Grip," in 2021 IEEE International Conference on Robotics and Automation (ICRA) (IEEE), 3707-3713. doi:10.1109/ ICRA48506.2021.9561744

Guidali, M., Duschau-Wicke, A., Broggi, S., Klamroth-Marganska, V., Nef, T., and Riener, R. (2011). A Robotic System to Train Activities of Daily Living in a Virtual Environment. Med. Biol. Eng. Comput. 49, 1213-1223. doi:10.1007/ s11517-011-0809-0

Gull, M. A., Bai, S., and Bak, T. (2020). A Review on Design of Upper Limb Exoskeletons. Robotics 9, 16-35. doi:10.3390/robotics9010016

Hill, P. W., Wolbrecht, E. T., and Perry, J. C. (2019). "Gravity Compensation of an Exoskeleton Joint Using Constant-Force Springs," in IEEE International Conference on Rehabilitation Robotics, 311-316. doi:10.1109/ ICORR.2019.8779422

Hogan, N. (1985). Impedance Control: An Approach to Manipulation: Part I-Theory. J. Dynamic Syst. Meas. Control Trans. ASME. 107, 1-7. doi:10.1115/1.3140702

Hong, K.-S., Ghafoor, U., and Khan, M. J. (2020). Brain-Machine Interfaces Using Functional Near-Infrared Spectroscopy: a Review. Artif. Life Robotics 25, 204-218. doi:10.1007/s10015-020-00592-9

Howlett, O. A., Lannin, N. A., Ada, L., and Mckinstry, C. (2015). Functional Electrical Stimulation Improves Activity After Stroke: A Systematic Review
With Meta-Analysis. Arch. Phys. Med. Rehabil. 96, 934-943. doi:10.1016/ J.APMR.2015.01.013

Huang, V. S., and Krakauer, J. W. (2009). Robotic Neurorehabilitation: A Computational Motor Learning Perspective. J. Neuroengineering Rehabil. 6, 5. doi:10.1186/1743-0003-6-5

Iandolo, R., Marini, F., Semprini, M., Laffranchi, M., Mugnosso, M., Cherif, A. et al. (2019). Perspectives and Challenges in Robotic Neurorehabilitation. Appl. Sci. 9, 3183. doi:10.3390/app9153183

[Dataset] Israely, S., and Carmeli, E. (2016). Error Augmentation as a Possible Technique for Improving Upper Extremity Motor Performance After a Stroke A Systematic Review. Top. Stroke Rehabil. 23, 116-125. doi:10.1179/ 1945511915Y.0000000007

[Dataset] Jarrassé, N. l., Proietti, T., Crocher, V., Robertson, J., Sahbani, A., Morel, G., et al. (2014). Robotic Exoskeletons: A Perspective for the Rehabilitation of Arm Coordination in Stroke Patients. Front. Hum. Neurosci. 8, 947. doi:10.3389/fnhum.2014.00947

Just, F., Baur, K., Riener, R., Klamroth-Marganska, V., and Rauter, G. (2016). "Online Adaptive Compensation of the ARMin Rehabilitation Robot," in Proceedings of the IEEE RAS and EMBS International Conference on Biomedical Robotics and Biomechatronics, 747-752. doi:10.1109/ BIOROB.2016.7523716

Just, F., Özen, Ö., Bösch, P., Bobrovsky, H., Klamroth-Marganska, V., Riener, R., et al. (2018). Exoskeleton Transparency: Feed-Forward Compensation vs. Disturbance Observer. At-Automatisierungstechnik. 66, 1014-1026. doi:10.1515/auto-2018-0069

Just, F., Özen, Ö., Tortora, S., Klamroth-Marganska, V., Riener, R., and Rauter, G. (2020). Human Arm Weight Compensation in Rehabilitation Robotics: Efficacy of Three Distinct Methods. J. Neuroengineering Rehabil. 17, 1-17. doi:10.1186/ s12984-020-0644-3

Just, F., Ozen, O., Tortora, S., Riener, R., and Rauter, G. (2017). "Feedforward Model Based Arm Weight Compensation with the Rehabilitation Robot ARMin," in IEEE International Conference on Rehabilitation Robotics, 72-77. doi:10.1109/ICORR.2017.8009224

Kahn, L. E., Lum, P. S., Rymer, W. Z., and Reinkensmeyer, D. J. (2006a). RobotAssisted Movement Training for the Stroke-Impaired Arm: Does it Matter what the Robot Does? J. Rehabil. Res. Development 43, 619-629. doi:10.1682/ JRRD.2005.03.0056

Kahn, L. E., Zygman, M. L., Rymer, W. Z., and Reinkensmeyer, D. J. (2006b). Robot-Assisted Reaching Exercise Promotes Arm Movement Recovery in Chronic Hemiparetic Stroke: A Randomized Controlled Pilot Study. J. Neuroengineering Rehabil. 3, 1-13. doi:10.1186/1743-0003-3-12

Keemink, A. Q., van der Kooij, H., and Stienen, A. H. (2018). Admittance Control for Physical Human-Robot Interaction. Int. J. Robotics Res. 37, 1421-1444. doi: $10.1177 / 0278364918768950$

Khalil, W., and Dombre, E. (2002). Compliant Motion Control. Model. Identification Control. Robots. 377-393. doi:10.1016/B978-190399666-9/ 50015-4

Khan, H., Naseer, N., Yazidi, A., Eide, P. K., Hassan, H. W., and Mirtaheri, P. (2021). Analysis of Human Gait Using Hybrid EEG-fNIRS-Based BCI System: A Review. Front. Hum. Neurosci. 14, 605. doi:10.3389/ fnhum.2020.613254

Kim, B., and Deshpande, A. D. (2015). "Controls for the Shoulder Mechanism of an Upper-Body Exoskeleton for Promoting Scapulohumeral Rhythm," in IEEE International Conference on Rehabilitation Robotics, 538-542. doi:10.1109/ ICORR.2015.7281255

Kim, B., and Deshpande, A. D. (2017). An Upper-Body Rehabilitation Exoskeleton Harmony With an Anatomical Shoulder Mechanism: Design, Modeling, Control, and Performance Evaluation. Int. J. Robotics Res. 36, 414-435. doi:10.1177/0278364917706743

Krakauer, J. W. (2006). Motor Learning: its Relevance to Stroke Recovery and Neurorehabilitation. Curr. Opin. Neurol. 19, 84-90. doi:10.1097/ 01.wco.0000200544.29915.cc

[Dataset] Krebs, H. I., Palazzolo, J. J., Dipietro, L., Ferraro, M., Krol, J., Rannekleiv, K., et al. (2003). Rehabilitation Robotics: Performance-Based Progressive Robot-Assisted Therapy. Autonomous Robots. 15, 7-20. doi:10.1023/A: 1024494031121

Kumar, S., Wöhrle, H., Trampler, M., Simnofske, M., Peters, H., Mallwitz, M., et al. (2019). Modular Design and Decentralized Control of the RECUPERA 
Exoskeleton for Stroke Rehabilitation. Appl. Sci. 9, 626. doi:10.3390/ app9040626

Kyoungchul, K., Moon, H., Jeon, D., and Tomizuka, M. (2010). Control of an Exoskeleton for Realization of Aquatic Therapy Effects. IEEE/ASME Trans. Mechatron. 15, 191-200. doi:10.1109/TMECH.2010.2041243

Langhorne, P., Coupar, F., and Pollock, A. (2009). Motor Recovery After Stroke: a Systematic Review. Lancet Neurol. 8, 741-754. doi:10.1016/S1474-4422(09) 70150-4

Lin, C.-H., Lien, W.-M., Wang, W.-W., Chen, S.-H., Lo, C.-H., Lin, S.-Y., et al. (2014). "NTUH-II Robot Arm With Dynamic Torque Gain Adjustment Method for Frozen Shoulder Rehabilitation," in 2014 IEEE/RSJ International Conference on Intelligent Robots and Systems (IEEE), 3555-3560. doi:10.1109/ IROS.2014.6943059

Lin, Y., Qu, Q., Lin, Y., He, J., Zhang, Q., Wang, C., et al. (20212021). Customizing Robot-Assisted Passive Neurorehabilitation Exercise Based on Teaching Training Mechanism. Biomed. Res. Int. 2021, 1-10. doi:10.1155/2021/9972560

Lo, H. S., and Xie, S. Q. (2012). Exoskeleton Robots for Upper-Limb Rehabilitation: State of the Art and Future Prospects. Med. Eng. Phys. 34, 261-268. doi:10.1016/ j.medengphy.2011.10.004

Lum, P. S., Burgar, C. G., Loos, M. V. d., Shor, P. C., Majmundar, M., and Yap, R. (2006). MIME Robotic Device for Upper-Limb Neurorehabilitation in Subacute Stroke Subjects: A Follow-Up Study. J. Rehabil. Res. Development. 43, 631-642. doi:10.1682/JRRD.2005.02.0044

Maciejasz, P., Eschweiler, J., Gerlach-Hahn, K., Jansen-Troy, A., and Leonhardt, S. (2014). A Survey on Robotic Devices for Upper Limb Rehabilitation. J. Neuroengineering Rehabil. 11, 3. doi:10.1186/1743-0003-11-3

Marchal-Crespo, L., and Reinkensmeyer, D. J. (2009). Review of Control Strategies for Robotic Movement Training After Neurologic Injury. J. Neuroengineering Rehabil. 6. doi:10.1186/1743-0003-6-20

Masiero, S., Celia, A., Rosati, G., and Armani, M. (2007). Robotic-Assisted Rehabilitation of the Upper Limb After Acute Stroke. Arch. Phys. Med. Rehabil. 88, 142-149. doi:10.1016/j.apmr.2006.10.032

Mehdi, H., and Boubaker, O. (2012). Stiffness and Impedance Control Using Lyapunov Theory for Robot-Aided Rehabilitation. Int. J. Soc. Robotics 4, 107-119. doi:10.1007/s12369-011-0128-5

Mehrholz, J. (2019). Is Electromechanical and Robot-Assisted Arm Training Effective for Improving Arm Function in People Who Have Had a Stroke? Am. J. Phys. Med. Rehabil. 98, 339-340. doi:10.1097/ PHM.0000000000001133

Miao, Q., Zhang, M., Cao, J., and Xie, S. Q. (2018). Reviewing High-Level Control Techniques on Robot-Assisted Upper-Limb Rehabilitation. Adv. Robotics. 32, 1253-1268. doi:10.1080/01691864.2018.1546617

Moubarak, S., Pham, M. T., Moreau, R., and Redarce, T. (2010). "Gravity Compensation of an Upper Extremity Exoskeleton," in 2010 Annual International Conference of the IEEE Engineering in Medicine and Biology (IEEE), 4489-4493. doi:10.1109/IEMBS.2010.5626036

Nef, T., Guidali, M., and Riener, R. (2009). ARMin III - Arm Therapy Exoskeleton With an Ergonomic Shoulder Actuation. Appl. Bionics Biomech. 6, 127-142. doi:10.1080/11762320902840179

Nef, T., and Lum, P. (2009). Improving Backdrivability in Geared Rehabilitation Robots. Med. Biol. Eng. Comput. 47, 441-447. doi:10.1007/s11517-0090437-0

Nef, T., Mihelj, M., and Riener, R. (2007). ARMin: A Robot for Patient-Cooperative Arm Therapy. Med. Bio Eng. Comput. 45, 887-900. doi:10.1007/s11517-0070226-6

Nicolas-Alonso, L. F., and Gomez-Gil, J. (2012). Brain Computer Interfaces, a Review. Sensors. 12, 1211-1279. doi:10.3390/s120201211

Noda, T., Sugimoto, N., Furukawa, J., Sato, M.-a., Hyon, S.-H., and Morimoto, J. (2012). "Brain-controlled Exoskeleton Robot for BMI Rehabilitation," in IEEE-RAS International Conference on Humanoid Robots, 21-27. doi:10.1109/HUMANOIDS.2012.6651494

Nordin, N., Xie, S., and Wünsche, B. (2014). Assessment of Movement Quality in Robot- Assisted Upper Limb Rehabilitation after Stroke: A Review. J. NeuroEngineering Rehabil. 11, 137. doi:10.1186/1743-0003-11-137

Novak, D., and Riener, R. (2013). "Enhancing Patient Freedom in Rehabilitation Robotics Using Gaze-Based Intention Detection," in 2013 IEEE 13th International Conference on Rehabilitation Robotics (ICORR) (IEEE), 1-6. doi:10.1109/ICORR.2013.6650507
Oldewurtel, F., Mihelj, M., Nef, T., and Riener, R. (2007). "Patient-Cooperative Control Strategies for Coordinated Functional Arm Movements," in 2007 European Control Conference, ECC 2007 (IEEE), 2527-2534. doi:10.23919/ ECC.2007.7068298

Oliveira, A. C. d., Warburton, K., Sulzer, J. S., and Deshpande, A. D. (2019). "Effort Estimation in Robot-Aided Training With a Neural Network," in Proceedings IEEE International Conference on Robotics and Automation, 563-569. doi:10.1109/ICRA.2019.8794281

Ostadabbas, S., Housley, S. N., Sebkhi, N., Richards, K., Wu, D., Zhang, Z., et al. (2016). Tongue-controlled Robotic Rehabilitation: A Feasibility Study in People With Stroke. J. Rehabil. Res. Dev. 53, 989-1006. doi:10.1682/JRRD.2015.06.0122

Ott, C., Mukherjee, R., and Nakamura, Y. (2010). "Unified Impedance and Admittance Control," in Proceedings - IEEE International Conference on Robotics and Automation (IEEE), 554-561. doi:10.1109/ROBOT.2010.5509861

Patton, J. L., Stoykov, M. E., Kovic, M., and Mussa-Ivaldi, F. A. (2006). Evaluation of Robotic Training Forces that Either Enhance or Reduce Error in Chronic Hemiparetic Stroke Survivors. Exp. Brain Res. 168, 368-383. doi:10.1007/ s00221-005-0097-8

Pérez-Ibarra, J. C., Siqueira, A. A. G., Silva-Couto, M. A., De Russo, T. L., and Krebs, H. I. (2019). Adaptive Impedance Control Applied to Robot-Aided Neuro-Rehabilitation of the Ankle. IEEE Robot. Autom. Lett. 4, 185-192. doi:10.1109/LRA.2018.2885165

Perry, J. C., Maura, R., Bitikofer, C. K., and Wolbrecht, E. T. (2018). BLUE SABINO: Development of a Bilateral Exoskeleton Instrument for Comprehensive Upper-Extremity Neuromuscular Assessment. Biosyst. Biorobotics. 21, 493-497. doi:10.1007/978-3-030-01845-0_99

Péter, O., Fazekas, G., Zsiga, K., and Dénes, Z. (2011). Robot-Mediated Upper Limb Physiotherapy. Int. J. Rehabil. Res. 34, 196-202. doi:10.1097/ MRR.0b013e328346e8ad

Pignolo, L., Lucca, L. F., Basta, G., Serra, S., Pugliese, M. E., Sannita, W. G., et al. (2016). A New Treatment in the Rehabilitation of the Paretic Upper Limb After Stroke: the ARAMIS Prototype and Treatment Protocol. Ann. Ist Super Sanita. 52, 301-308. doi:10.4415/ANN16022510.4415/ANN_16_02_25

Pignolo, L., Dolce, G., Basta, G., Lucca, L. F., Serra, S., and Sannita, W. G. (2012). "Upper Limb Rehabilitation After Stroke: ARAMIS a "Robo-Mechatronic" Innovative Approach and Prototype," in 2012 4th IEEE RAS \& EMBS International Conference on Biomedical Robotics and Biomechatronics (BioRob) (IEEE), 1410-1414. doi:10.1109/BioRob.2012.6290868

Pirondini, E., Coscia, M., Marcheschi, S., Roas, G., Salsedo, F., Frisoli, A., et al. (2016). Evaluation of the Effects of the Arm Light Exoskeleton on Movement Execution and Muscle Activities: a Pilot Study on Healthy Subjects. J. Neuroengineering Rehabil. 13, 9. doi:10.1186/s12984-016-0117-x

Proietti, T., Crocher, V., Roby-Brami, A., and Jarrasse, N. (2016). Upper-Limb Robotic Exoskeletons for Neurorehabilitation: A Review on Control Strategies. IEEE Rev. Biomed. Eng. 9, 4-14. doi:10.1109/RBME.2016.2552201

Proietti, T., Guigon, E., Roby-Brami, A., and Jarrassé, N. (2017). Modifying UpperLimb Inter-Joint Coordination in Healthy Subjects by Training With a Robotic Exoskeleton. J. Neuroengineering Rehabil. 14, 1-19. doi:10.1186/S12984-0170254-X

Proietti, T., Jarrasse, N., Roby-Brami, A., and Morel, G. (2015). “Adaptive Control of a Robotic Exoskeleton for Neurorehabilitation,” in International IEEE/EMBS Conference on Neural Engineering, NER (IEEE), 803-806. doi:10.1109/ NER.2015.7146745

Puchinger, M., Kurup, N. B. R., Keck, T., Zajc, J., Russold, M. F., and Gfohler, M. (2018). "The Retrainer Light-Weight Arm Exoskeleton: Effect of Adjustable Gravity Compensation on Muscle Activations and Forces," in Proceedings of the IEEE RAS and EMBS International Conference on Biomedical Robotics and Biomechatronics, 396-401. doi:10.1109/BIOROB.2018.8487218

Ragonesi, D., Agrawal, S. K., Sample, W., and Rahman, T. (2013). Quantifying Anti-gravity Torques for the Design of a Powered Exoskeleton. IEEE Trans. Neural Syst. Rehabil. Eng. 21, 283-288. doi:10.1109/TNSRE.2012.2222047

Rehmat, N., Zuo, J., Meng, W., Liu, Q., Xie, S. Q., and Liang, H. (2018). Upper Limb Rehabilitation Using Robotic Exoskeleton Systems: a Systematic Review. Int. J. Intell. Robot Appl. 2, 283-295. doi:10.1007/s41315-018-0064-8

Reinkensmeyer, D. J., Burdet, E., Casadio, M., Krakauer, J. W., Kwakkel, G., Lang, C. E., et al. (2016). Computational Neurorehabilitation: Modeling Plasticity and Learning to Predict Recovery. J. Neuroengineering Rehabil. 13, 42. doi:10.1186/ s12984-016-0148-3 
Rodgers, H., Bosomworth, H., Krebs, H. I., van Wijck, F., Howel, D., Wilson, N., et al. (2019). Robot Assisted Training for the Upper Limb After Stroke (RATULS): a Multicentre Randomised Controlled Trial. The Lancet 394, 51-62. doi:10.1016/S0140-6736(19)31055-4

Sanchez, R. J., Wolbrecht, E., Smith, R., Liu, J., Rao, S., Cramer, S., et al. (2005). “A Pneumatic Robot for Re-Training Arm Movement After Stroke: Rationale and Mechanical Design," in Proceedings of the 2005 IEEE 9th International Conference on Rehabilitation Robotics 2005, 500-504. doi:10.1109/ ICORR.2005.1501151

Schumacher, M., Wojtusch, J., Beckerle, P., and von Stryk, O. (2019). An Introductory Review of Active Compliant Control. Robotics Autonomous Syst. 119, 185-200. doi:10.1016/j.robot.2019.06.009

Shadmehr, R., Smith, M. A., and Krakauer, J. W. (2010). Error Correction, Sensory Prediction, and Adaptation in Motor Control. Annu. Rev. Neurosci. 33, 89-108. doi:10.1146/annurev-neuro-060909-153135

Shen, Y., Ma, J., Dobkin, B., and Rosen, J. (2018). "Asymmetric Dual Arm Approach for Post Stroke Recovery of Motor Functions Utilizing the EXOUL8 Exoskeleton System: A Pilot Study," in Proceedings of the Annual International Conference of the IEEE Engineering in Medicine and Biology Society, Honolulu, HI, USA (EMBS (Institute of Electrical and Electronics Engineers Inc), 1701-1707. doi:10.1109/EMBC.2018.8512665

Shen, Y., Sun, J., Ma, J., and Rosen, J. (2019). "Admittance Control Scheme Comparison of EXO-UL8: A Dual-Arm Exoskeleton Robotic System," in 2019 IEEE 16th International Conference on Rehabilitation Robotics (ICORR) (IEEE), 611-617. doi:10.1109/ICORR.2019.8779545

Soltani-Zarrin, R., Zeiaee, A., Eib, A., Langari, R., Robson, N., and Tafreshi, R. (2017a). "TAMU CLEVERarm: A Novel Exoskeleton for Rehabilitation of Upper Limb Impairments," in 2017 International Symposium on Wearable Robotics and Rehabilitation, WeRob 2017 (Institute of Electrical and Electronics Engineers Inc.), 1-2. doi:10.1109/WEROB.2017.8383844

Soltani-Zarrin, R., Zeiaee, A., Langari, R., and Robson, N. (2017b). "Reference Path Generation for Upper-Arm Exoskeletons Considering Scapulohumeral Rhythms," in IEEE International Conference on Rehabilitation Robotics, London, UK, 753-758. doi:10.1109/ICORR.2017.8009338

Song, Z., Guo, S., Pang, M., Zhang, S., Xiao, N., Gao, B., et al. (2014). Implementation of Resistance Training Using an Upper-Limb Exoskeleton Rehabilitation Device for Elbow Joint. J. Med. Biol. Eng. 34, 188-196. doi:10.5405/jmbe.1337

Stroppa, F., Loconsole, C., Marcheschi, S., and Frisoli, A. (2017a). A Robot-Assisted Neuro-Rehabilitation System for Post-Stroke Patients' Motor Skill Evaluation with ALEx Exoskeleton. Biosyst. Biorobotics 15, 501-505. doi:10.1007/978-3319-46669-9_83

Stroppa, F., Marcheschi, S., Mastronicola, N., Loconsole, C., and Frisoli, A. (2017b). "Online Adaptive Assistance Control in Robot-Based Neurorehabilitation Therapy," in IEEE International Conference on Rehabilitation Robotics (IEEE Computer Society), 628-633. doi:10.1109/ ICORR.2017.8009318

Stroppa, F., Loconsole, C., Marcheschi, S., Mastronicola, N., and Frisoli, A. (2018). "An Improved Adaptive Robotic Assistance Methodology for Upper-Limb Rehabilitation," in Lecture Notes in Computer Science (including subseries Lecture Notes in Artificial Intelligence and Lecture Notes in Bioinformatics) (Cham: Springer), 513-525. doi:10.1007/978-3-319-93399-3_44

Trigili, E., Crea, S., Moise, M., Baldoni, A., Cempini, M., Ercolini, G., et al. (2019). Design and Experimental Characterization of a Shoulder-Elbow Exoskeleton with Compliant Joints for Post-Stroke Rehabilitation. IEEE/ASME Trans. Mechatron. 24, 1485-1496. doi:10.1109/TMECH.2019.2907465

Vallery, H., Veneman, J., van Asseldonk, E., Ekkelenkamp, R., Buss, M., and van Der Kooij, H. (2008). Compliant Actuation of Rehabilitation Robots. IEEE Robot. Automat. Mag. 15, 60-69. doi:10.1109/MRA.2008.927689

Van Delden, A. E. Q., Peper, C. E., Kwakkel, G., and Beek, P. J. (2012). A Systematic Review of Bilateral Upper Limb Training Devices for Poststroke Rehabilitation. Stroke Res. Treat. 2012, 1-17. doi:10.1155/2012/972069

Veerbeek, J. M., Langbroek-Amersfoort, A. C., Van Wegen, E. E. H., Meskers, C. G. M., and Kwakkel, G. (2017). Effects of Robot-Assisted Therapy for the Upper Limb after Stroke. Neurorehabil. Neural Repair. 31, 107-121. doi:10.1177/ 1545968316666957

Villani, L., and De Schutter, J. (2008). Force Control. Springer Handbook of Robotics., 161-185. doi:10.1007/978-3-540-30301-5_8
Wang, J., and Barry, O. R. (2021). Inverse Optimal Robust Adaptive Controller for Upper Limb Rehabilitation Exoskeletons with Inertia and Load Uncertainties. IEEE Robot. Autom. Lett. 6, 2171-2178. doi:10.1109/LRA.2021.3061361

Weiss, P., Zenker, P., and Maehle, E. (2012). "Feed-Forward Friction and Inertia Compensation for Improving Backdrivability of Motors," in 2012 12th International Conference on Control, Automation, Robotics and Vision, ICARCV, 288-293. doi:10.1109/ICARCV.2012.6485173

Winter, D. A. (2009). Kinetics: Forces and Moments of Force. Biomech. Mot. Control. Hum. Movement., 107-138. doi:10.1002/9780470549148.ch5

Wolbrecht, E. T., Chan, V., Reinkensmeyer, D. J., and Bobrow, J. E. (2008). Optimizing Compliant, Model-Based Robotic Assistance to Promote Neurorehabilitation. IEEE Trans. Neural Syst. Rehabil. Eng. 16, 286-297. doi:10.1109/TNSRE.2008.918389

Wright, Z. A., Lazzaro, E., Thielbar, K. O., Patton, J. L., and Huang, F. C. (2018). Robot Training With Vector Fields Based on Stroke Survivors' Individual Movement Statistics. IEEE Trans. Neural Syst. Rehabil. Eng. 26, 307-323. doi:10.1109/TNSRE.2017.2763458

Wright, Z. A., Patton, J. L., Huang, F. C., and Lazzaro, E. (2015). "Evaluation of Force Field Training Customized According to Individual Movement Deficit Patterns," in IEEE International Conference on Rehabilitation Robotics, 193-198. doi:10.1109/ICORR.2015.7281198

Wu, J., Cheng, H., Zhang, J., Yang, S., and Cai, S. (2021). Robot-Assisted Therapy for Upper Extremity Motor Impairment After Stroke: A Systematic Review and Meta-Analysis. Phys. Ther. 101, pzab010. doi:10.1093/ptj/pzab010

Wu, K.-Y., Su, Y.-Y., Yu, Y.-L., Lin, C.-H., and Lan, C.-C. (2019). A 5-Degrees-OfFreedom Lightweight Elbow-Wrist Exoskeleton for Forearm Fine-Motion Rehabilitation. IEEE/ASME Trans. Mechatron. 24, 2684-2695. doi:10.1109/ TMECH.2019.2945491

Wu, Q., Wang, X., Chen, B., and Wu, H. (2018a). Development of a MinimalIntervention-Based Admittance Control Strategy for Upper Extremity Rehabilitation Exoskeleton. IEEE Trans. Syst. Man. Cybern. Syst. 48, 1005-1016. doi:10.1109/TSMC.2017.2771227

Wu, Q., Wang, X., Chen, B., and Wu, H. (2018b). Patient-Active Control of a Powered Exoskeleton Targeting Upper Limb Rehabilitation Training. Front. Neurol. 9, 817. doi:10.3389/fneur.2018.00817

Xu, G., Song, A., and Li, H. (2011). Control System Design for an Upper-Limb Rehabilitation Robot. Adv. Robotics 25, 229-251. doi:10.1163/ 016918610 X538561

Zeiaee, A., Soltani-Zarrin, R., Langari, R., and Tafreshi, R. (2019). Kinematic Design Optimization of an Eight Degree-Of-Freedom Upper-Limb Exoskeleton. Robotica 37, 2073-2086. doi:10.1017/S0263574719001085

Zhang, L., Guo, S., and Sun, Q. (2020). An Assist-As-Needed Controller for Passive, Assistant, Active, and Resistive Robot-Aided Rehabilitation Training of the Upper Extremity. Appl. Sci. 11, 340. doi:10.3390/app11010340

Zhuang, Y., Yao, S., Ma, C., and Song, R. (2019). Admittance Control Based on EMG-Driven Musculoskeletal Model Improves the Human-Robot Synchronization. IEEE Trans. Ind. Inf. 15, 1211-1218. doi:10.1109/ TII.2018.2875729

Conflict of Interest: SD, AP, FB, and MG hold shares in AGADE Srl, Milano, Italy.

The remaining authors declare that the research was conducted in the absence of any commercial or financial relationships that could be construed as a potential conflict of interest.

Publisher's Note: All claims expressed in this article are solely those of the authors and do not necessarily represent those of their affiliated organizations, or those of the publisher, the editors and the reviewers. Any product that may be evaluated in this article, or claim that may be made by its manufacturer, is not guaranteed or endorsed by the publisher.

Copyright (c) 2021 Dalla Gasperina, Roveda, Pedrocchi, Braghin and Gandolla. This is an open-access article distributed under the terms of the Creative Commons Attribution License (CC BY). The use, distribution or reproduction in other forums is permitted, provided the original author(s) and the copyright owner $(s)$ are credited and that the original publication in this journal is cited, in accordance with accepted academic practice. No use, distribution or reproduction is permitted which does not comply with these terms. 